\title{
Calcul tridimensionnel de la résultante d'une chaîne de cotes en cotation ISO, en vue d'un calcul probabiliste
}

\author{
BeRnARD Anselmetti ${ }^{\mathrm{a}}$ \\ Univ. Paris Sud, IUT de Cachan, LURPA, ENS de Cachan, 61 avenue du président Wilson, 94235 Cachan Cedex, France
}

Reçu le 15 mars 2007, accepté le 7 juin 2007

\begin{abstract}
Résumé - En cotation fonctionnelle, il est nécessaire de calculer l'influence de tous les défauts admis sur les pièces par leur cotation selon les normes ISO pour vérifier le respect des exigences fonctionnelles. La méthode CLIC (Cotation en Localisation avec Influence des Contacts) comprend des algorithmes de choix des spécifications, une méthode originale et complète de calcul 3D de la résultante dite « des droites d'analyse », au pire des cas et en statistique, et une méthode de synthèse des tolérances. Cet article présente l'influence des défauts d'une jonction sur l'exigence et la décomposition d'une exigence complexe en sous-exigences pour le cumul statistique des influences. Une application tridimensionnelle statistique est proposée avec une présentation de la feuille de calcul sous Microsoft EXCEL.
\end{abstract}

Mots clés : Cotation ISO / cotation statistique / tolérancement 3D / torseur des petits déplacements / droites d'analyse

\begin{abstract}
Functional tolerancing requires calculating the influence of all the deviations admitted on the parts by their definition drawings according to ISO standards' to check the respect of the functional requirements. The method CLIC (Tolerancing in Localization with Influence of the Contacts) includes algorithms of choice of the specifications, an original and complete 3D method of calculation of the result based on the "analysis lines", at worse cases and in statistic, and a method of synthesis of the tolerances. This paper presents the influence of the deviations of a junction on the requirement and the decomposition of a complex requirement in sub-requirements. A three-dimensional statistical application is proposed with a presentation of the worksheet Microsoft EXCEL.
\end{abstract}

Key words: ISO tolerancing / statistical tolerancing / three-dimensional tolerancing / small displacement torsor / analysis lines

\section{Besoin du calcul 3D statistique}

\subsection{Principe de la cotation fonctionnelle}

La cotation fonctionnelle en utilisant les normes ISO de cotation est devenue une obligation pour les entreprises, dans le contexte des échanges internationaux. La méthode CLIC développée au laboratoire [1] permet aujourd'hui d'élaborer cette cotation fonctionnelle avec une approche réellement tridimensionnelle. La méthode « des droites d'analyse » est utilisée pour calculer les cumuls des défauts des différentes pièces. Il s'agit d'établir, pour chaque exigence, une inéquation qui relie les dimensions nominales des pièces et les tolérances des spécifications, à la valeur limite de l'exigence. L'étape ultime de synthèse

\footnotetext{
a Auteur correspondant : anselm@lurpa.ens-cachan.fr
}

de tolérances optimise les valeurs nominales pour maximiser les tolérances. L'ensemble de cette démarche est formalisée dans une maquette informatique sous Microsoft EXCEL, en utilisant le langage VBA pour générer les formules de calcul du tableur et le solveur pour la synthèse des tolérances.

Le problème traité dans cet article est celui des chaînes de cotes tridimensionnelles qui font intervenir des défauts géométriques et des mobilités permises par des jeux. La figure 1 illustre des exigences externes complexes, provenant de la décomposition d'un mécanisme en sousensembles. Il faut donc établir les chaînes de cotes et spécifier chaque pièce pour établir la relation donnant l'écart résultant en fonction des tolérances des pièces influentes.

Plusieurs défauts angulaires et radiaux d'une même pièce peuvent notamment être influents sur une exigence, 


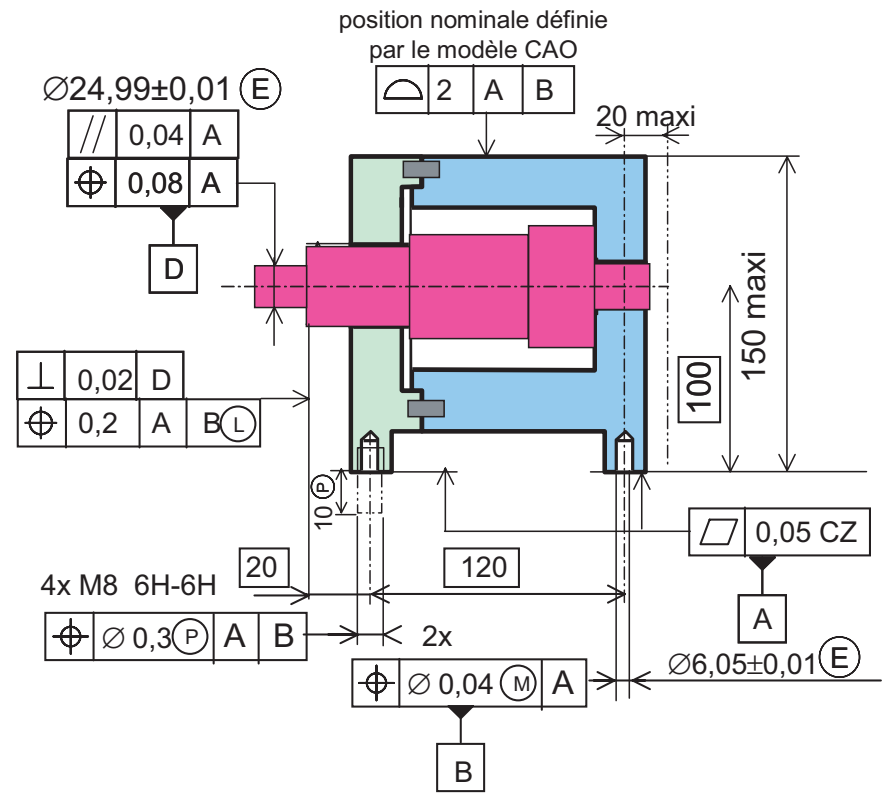

Fig. 1. Exemple d'exigences complexes à transférer.

ce qui rend impossible l'emploi d'une méthode statistique basée simplement sur la somme des variances associées à chaque tolérance. Cette difficulté a nécessité le développement d'une méthode particulière, pour pouvoir séparer le plus possible l'influence des défauts de chaque pièce.

\section{2 État de l'art industriel}

En entreprise, le calcul des chaînes de cotes classiques repose sur la traditionnelle somme vectorielle des cotes nominales et l'addition des tolérances des pièces influentes, ce qui limite son emploi aux chaînes de cotes unidirectionnelles, sans prendre en compte les effets angulaires. Dans ce contexte unidirectionnel, il n'y a qu'un seul maillon par pièce, le calcul statistique ne pose pas de problème.

Quelques applications spécifiques sous Microsoft EXCEL permettent des calculs radiaux au pire des cas, par exemple pour calculer les jeux dans des montages avec deux centreurs.

Dès que la résultante dépend d'effets angulaires ou de jeux radiaux, les calculs sont beaucoup plus complexes. Divers auteurs ont travaillé avec succès sur ce thème, notamment avec des matrices de rotation ou le torseur des petits déplacements pour traduire l'effet des trois translations et des trois rotations sur la résultante. Outre que les calculs ne sont pas simples, le résultat n'est pas facilement exprimable en fonction des tolérances ISO produites sur les dessins pour la synthèse des tolérances.

Le problème devient encore plus complexe lorsqu'il s'agit de faire un calcul statistique de la résultante car l'addition des variances nécessite que les variables soient indépendantes. Une des difficultés vient du fait que plusieurs défauts géométriques de la même pièce (donc non indépendants), peuvent être influents sur l'exigence. De plus, l'écart d'une surface est exprimé avec des paramètres angulaires ou de translation qui ne sont pas indépendants.

Pour contourner ce problème, divers logiciels utilisent la méthode de Monte Carlo qui effectue un tirage aléatoire de pièces avec défauts pour simuler l'assemblage. Deux difficultés majeures sont toutefois perçues : il est très difficile de générer les distributions aléatoires qui reproduisent les différentes dépendances entre les paramètres. D'autre part, comme il y a un grand nombre de paramètres, il faut un très grand nombre de tirages pour obtenir une bonne précision sur l'estimation des valeurs limites. De plus, la méthode de Monte Carlo ne donne pas directement la relation recherchée entre la résultante et les tolérances des pièces influentes, ce qui ne permet pas l'étape suivante de synthèse des tolérances.

Le travail original présenté dans cette communication a pour but d'établir, pour chaque exigence, une relation généralement linéaire au pire des cas ou quadratique en statistique, en fonction des tolérances portées sur le dessin. Le principe de la méthode dite « des droites d'analyse » consiste à étudier chaque jonction séparément pour déterminer son influence sur la position de la surface fonctionnelle terminale. Pour cela, les surfaces terminales sont discrétisées. La méthode tient compte des jeux, des contacts primaires, secondaires et tertiaires et permet de traiter des jonctions non-orthomorphes, sans approximation ou avec des approximations très raisonnables. Dans certains cas, il peut y avoir une légère combinatoire avec deux à six combinaisons par jonction.

\section{Principe du tolérancement 3D avec CLIC}

\subsection{Discrétisation des surfaces terminales}

L'exigence étudiée porte sur une surface terminale qui doit être discrétisée. Si l'exigence est une localisation d'un plan, le contour est discrétisé en plusieurs points fonctionnels $F i$ (Fig. 2a). Il faut étudier le déplacement de chaque point $F i$, dans la direction $\vec{f}$ normale au plan. Ce déplacement est noté $\mathrm{d}(F i, \vec{f})$. Pour une localisation de l'axe d'un cylindre avec une zone de tolérance définie par deux plans perpendiculaires à la direction $\vec{f}$, il suffit d'étudier le déplacement des deux extrémités de l'axe du cylindre (Fig. 2b). Si la zone de tolérance est cylindrique, la discrétisation est radiale (Fig. 2c). Si l'exigence est une orientation, il faut déterminer le déplacement angulaire maxi autour de la direction $\vec{f}$ noté a $(\Delta, \vec{f}), \Delta$ étant l'axe du cylindre ou une droite du plan (Fig. 2d). La résultante maxi sera obtenue en déterminant le plus grand des déplacements sur les différents points.

\subsection{Influence des jonctions}

Pour généraliser le problème, il faut être capable de traiter des assemblages avec des surfaces inclinées, des jonctions en série et en parallèle, des surfaces de contacts, 

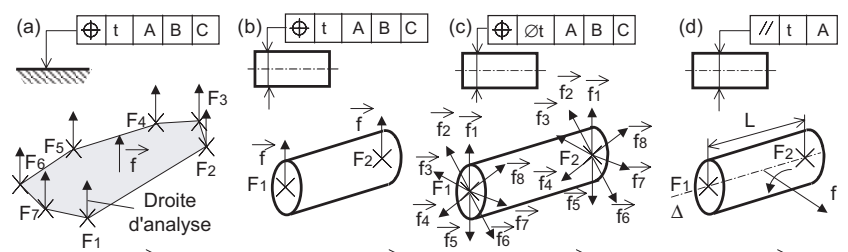

$r=\max [\mathrm{d}(\mathrm{Fi}, \overrightarrow{\mathrm{f}})]$ $r=\max [\mathrm{d}(\mathrm{F}$

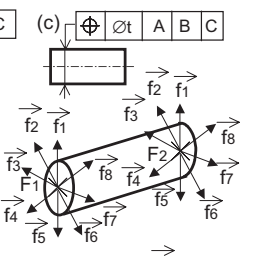

Fig. 2. Discrétisation des surfaces terminales.

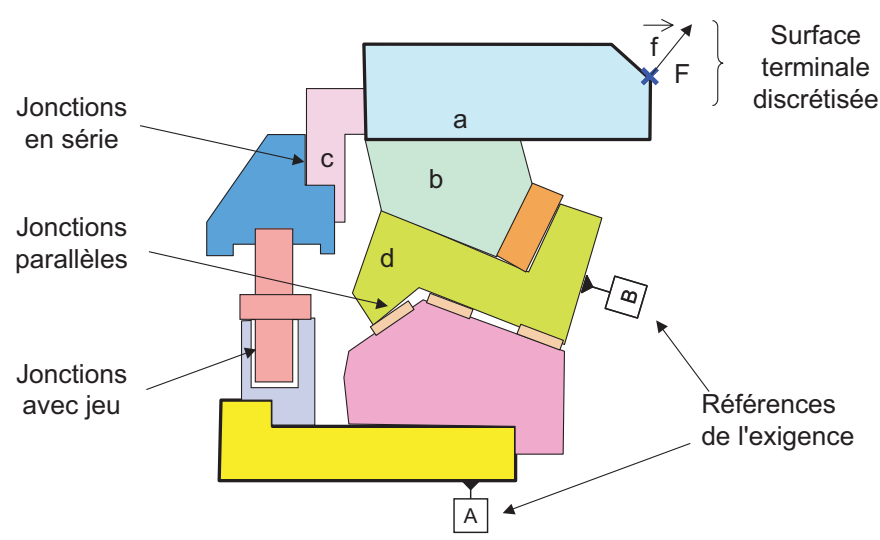

Fig. 3. Mécanisme 3D générique.

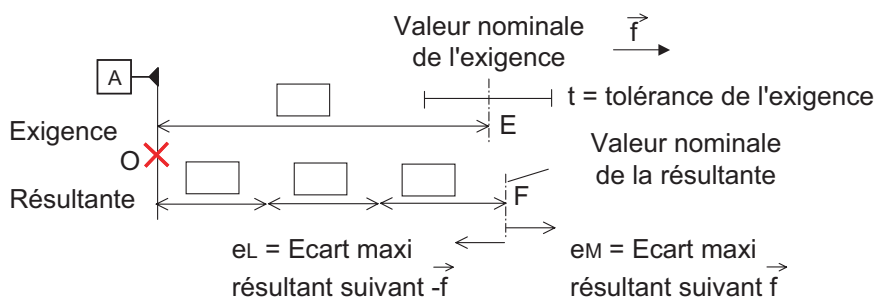

Fig. 4. Calcul des écarts à respecter.

des liaisons avec jeu ou avec serrage (Fig. 3). Le but est donc de calculer l'influence des écarts de chaque surface sur le déplacement du point $F$ dans la direction d'analyse $\vec{f}$, par rapport à un système de références.

\subsection{Base du calcul de l'inéquation à respecter}

Le principe du calcul est schématisé en unidirectionnel sur la figure 4. L'exigence impose que la surface terminale se trouve en $E$, à une position théorique décrite par des cotes encadrées par rapport au système de références défini sur des surfaces du mécanisme et dans une zone de tolérance de largeur $t$. La normale à cette surface fonctionnelle est $\vec{f}$. En pratique, la surface nominale sera positionnée en $F$ en fonction du cumul des cotes encadrées des pièces influentes et avec un écart maxi qui dépend des tolérances des pièces influentes. Les relations à respecter sont les suivantes :

$$
e_{\mathrm{L}}-\overrightarrow{E F} \cdot \vec{f} \leqslant t / 2 \quad \text { et } \quad \overrightarrow{E F} \cdot \vec{f}+e_{\mathrm{M}} \leqslant t / 2
$$

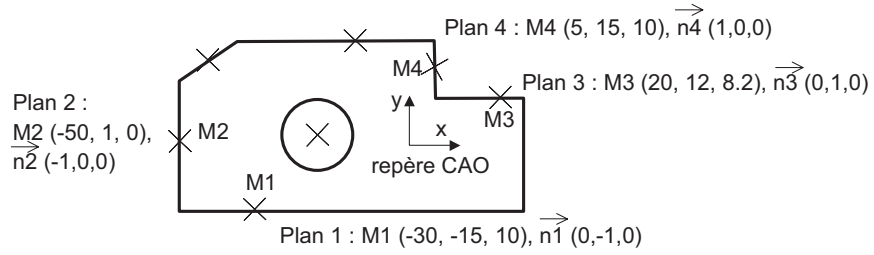

Fig. 5. Définition partielle du modèle nominal initial.

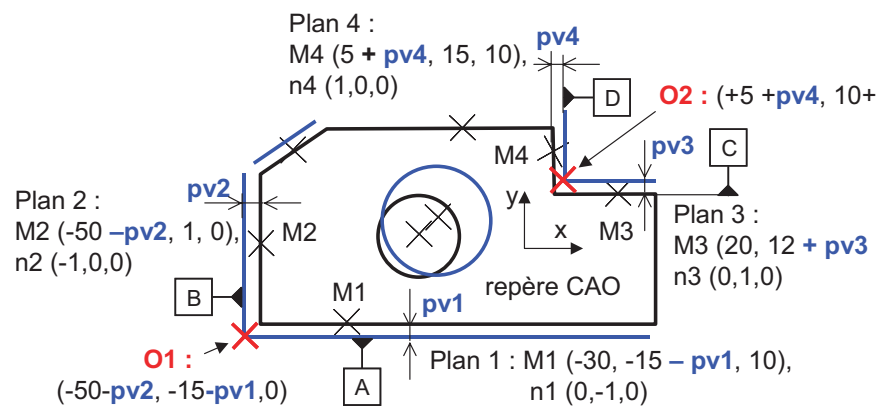

Fig. 6. Origines des repères sur le nominal paramétré.

\section{Variation du modèle nominal}

\subsection{Nominal initial}

Le modèle nominal est défini en B REP par des faces principalement planes ou cylindriques. Chaque surface est identifiée par un point et une normale dirigée hors matière, dans le repère $\mathrm{CAO}$ de la pièce (Fig. 5).

\subsection{Nominal paramétré}

Le but final étant d'optimiser les dimensions nominales des pièces, il faut paramétrer ce modèle nominal. Pour avoir des variables indépendantes et conserver la forme et la cohérence de la pièce, la méthode CLIC propose de faire un décalage de chaque surface d'une petite variation $p v$ qui sera de quelques centièmes de $\mathrm{mm}$, ce qui ne doit pas perturber les caractéristiques mécaniques de la pièce. Il est donc inutile d'introduire des contraintes d'épaisseurs mini de matière par exemple. Ces variations de position des surfaces sont générées de sorte que les propriétés de symétrie de la pièce soient conservées, grâce à une analyse complète de la topologie de la pièce.

La méthode CLIC impose de déclarer la gamme d'assemblage du mécanisme à l'aide des tableaux de mise en position de chaque pièce [2]. Un système de références est alors construit sur chaque groupe de surface de jonction. La figure 6 montre le système de références principal $A B$ avec son origine $\mathrm{O} 1$ et le système de références auxiliaires $\mathrm{CD}$ avec son origine $\mathrm{O} 2$. O1 et $\mathrm{O} 2$ sont donc définis dans le repère $\mathrm{CAO}$ de chaque pièce et en fonction des petites variations $p v 1, p v 2 \ldots$

\subsection{Position de tout point dans le repère de la base}

À partir des tableaux de mise en position, un algorithme simule l'assemblage de proche en proche en 


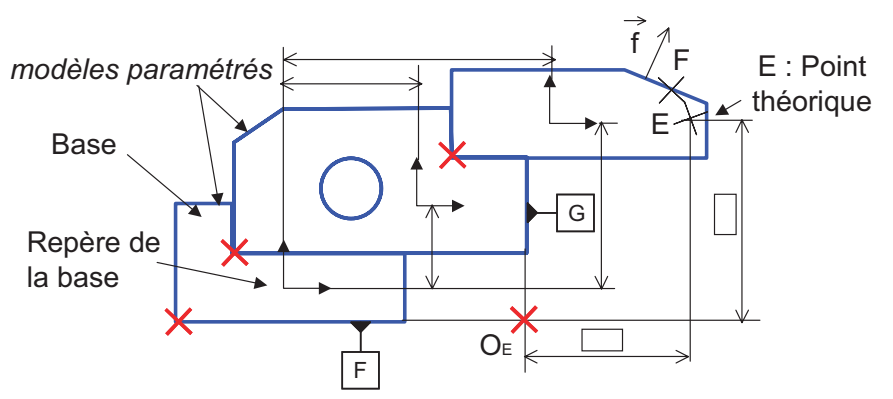

Fig. 7. Assemblage des pièces paramétrées.
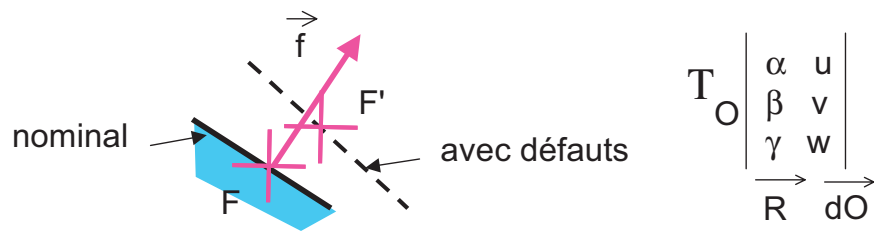

Fig. 8. Écart d'un point fonctionnel.

superposant les origines des systèmes de références (Fig. 7). Cette approche détermine la position de tous les repères $\mathrm{CAO}$ de chaque pièce par rapport au repère CAO de la base du mécanisme. Cela permet de connaître la position de toutes les surfaces dans le repère CAO de la base et de calculer par exemple l'origine du repère $\mathrm{OE}$ correspondant à l'exigence étudiée.

Dans l'environnement EXCEL, toutes ces positions sont calculées par des formules, en fonction des petites variations qui seront des variables inconnues du problème.

Dans la figure $7, E$ est la position théorique définie par l'exigence $\left(\overrightarrow{O_{\mathrm{E}} E}\right.$ est défini par les cotes encadrées). La contribution de l'écart du nominal est donc :

$$
\overrightarrow{E F} \cdot \vec{f}=\left(\overrightarrow{O_{\mathrm{E}} F}-\overrightarrow{O_{\mathrm{E}} E}\right) \cdot \vec{f}
$$

\section{Cumul des écarts dans une branche de mise en position}

\subsection{Influence des défauts et des jeux}

Il s'agit ici de déterminer l'écart maxi de la surface terminale par rapport à la surface nominale (Fig. 8). Le problème est tridimensionnel et doit prendre en compte les effets angulaires. On applique la méthode des torseurs des petits déplacements qui est une linéarisation du déplacement 3D rendue possible car les déplacements dus aux tolérances et aux jeux sont petits. L'écart dans la direction $\vec{f}$ est $\overrightarrow{F F^{\prime}} \cdot \vec{f}$. Cet écart sera noté $\mathrm{d}(F, \vec{f})$. On peut aussi étudier l'écart dans la direction opposée $\mathrm{d}(F,-\vec{f})$, qui n'est pas forcément identique à celui dans la direction $\vec{f}$.

Pour effectuer ce type de calcul, P. Bourdet $[3,4]$ utilise un calcul formel pour écrire le torseur des petits déplacements de la surface terminale en fonction de toutes les conditions de contact. La valeur maximale est trouvée en introduisant des contraintes représentant les tolérances
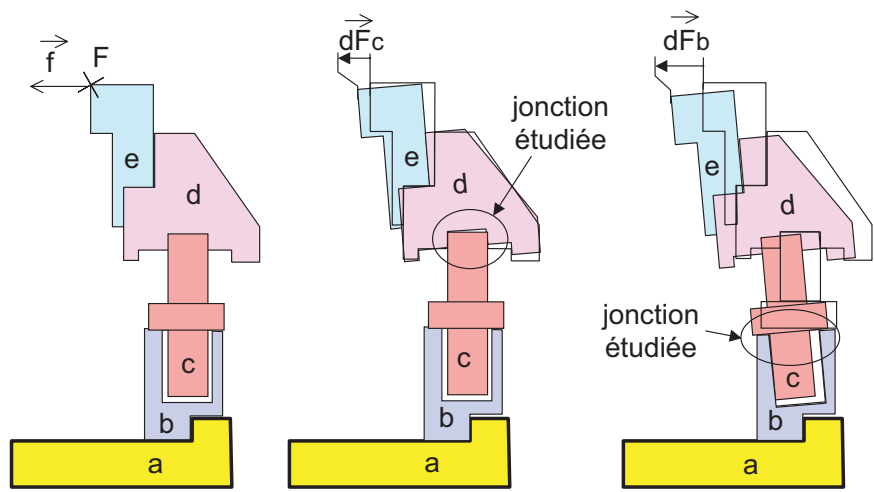

Fig. 9. Cumul des influences des jonctions.

de chaque pièce. La prise en compte des contacts secondaires et tertiaires impose une combinatoire. Le système obtenu est assez complexe, et n'est pas facile à simplifier pour faire la synthèse des tolérances.

P. Clozel utilise également le torseur des petits déplacements et une méthode matricielle, mais les liaisons sont simplifiées en adoptant une modélisation isostatique [5]. Cette approche permet de traiter de grands systèmes.

La méthode CLIC étudie le déplacement de chaque point de la surface terminale dans la direction d'analyse $\vec{f}$, en ajoutant successivement le déplacement dû à chaque jonction (Fig. 9). Durant ce calcul, toutes les pièces sont parfaites, seules les surfaces de la jonction étudiée ont des défauts par rapport aux systèmes de références principal de la pièce considérée.

Il est donc possible d'établir l'influence des défauts de la jonction étudiée sur la surface terminale en considérant que toutes les pièces entre la jonction et la surface terminale sont « soudées » en une seule pièce, ce qui revient à étudier une jonction entre deux pièces.

\subsection{Influence des défauts d'une jonction isostatique}

La méthode des droites d'analyse est une méthode originale qui peut être présentée dans un premier temps sur un assemblage simple de deux pièces, avec un montage isostatique (Fig. 10). Trois points P1, P2, P3 définissent un appui plan primaire, les points $\mathrm{S} 1$ et $\mathrm{S} 2$ définissent une linéique secondaire et un sixième point tertiaire $T$. Les trois faces planes sont orientées de manière quelconque (jonction non orthomorphe).

La mise en position étant isostatique, pour toute combinaison d'écart des 6 points d'appui, on peut calculer le déplacement du point $F$, à l'aide d'une simple combinaison linéaire, ce qui donne l'influence de chaque point. Cependant, pour calculer le déplacement maxi de $F$, il n'est pas suffisant de prendre la somme des influences maxi de chaque point, car les écarts des 6 points ne sont pas indépendants. Il en est de même pour le calcul statistique de $F$. Par exemple, selon le procédé de fabrication, il est parfois impossible d'avoir à la fois le point $\mathrm{P} 1$ le plus 


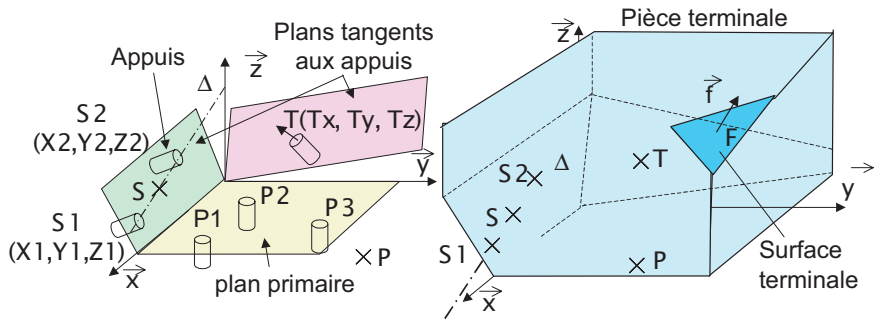

Fig. 10. Influence des défauts d'un montage isostatique.

haut possible et P2 le plus bas possible permis par leurs intervalles de tolérance.

Il est possible de calculer le déplacement du point $F$ à partir du déplacement de trois points particuliers appartenant respectivement aux plans de contact primaire, secondaire et tertiaire.

Pour simplifier cette démonstration, $z$ est normal au plan primaire, la direction $x$ est à l'intersection des plans primaire et secondaire. L'origine est à l'intersection des trois plans.

Le problème est de calculer : $\mathrm{d}(F, \vec{f})=\overrightarrow{\mathrm{d} F} \cdot \vec{f}$ avec $F(F x, F y, F z)$ et $\vec{f}(f x, f y, f z)$ (unitaire).

Les défauts des points d'appui induisent un petit déplacement du système de références partielles construit sur la pièce terminale. Ce déplacement est défini par la translation $(u, v, w)$ de l'origine et par une rotation $\vec{\Omega}$.

Le déplacement du point $F$ est :

$$
\overrightarrow{\mathrm{d} F} \cdot \vec{f}=u \cdot f x+v \cdot f y+w \cdot f z+\vec{\Omega} \cdot(\overrightarrow{O F} \wedge \vec{f})
$$

Si $T(T x, T y, T z)$ est le point de contact tertiaire de la pièce terminale avec son appui, on peut écrire :

$$
\overrightarrow{\mathrm{dT}} \cdot \vec{t}=u \cdot t x+v \cdot t y+w \cdot t z+\vec{\Omega} \cdot(\overrightarrow{O T} \wedge \vec{t})
$$

avec $\vec{t}(t x, t y, t z)$ On cherche un point $S$ de la pièce terminale appartenant à la droite passant par les deux points de contact secondaire :

$$
\overrightarrow{\mathrm{d} S} \cdot \vec{s}=v \cdot s y+w \cdot s z+\vec{\Omega} \cdot(\overrightarrow{O S} \wedge \vec{s}) \text { avec } \vec{s}(0, s y, s z)
$$
unitaire.

On cherche un point $P$ du plan de contact primaire avec $\vec{p}(0,0,1)$

$$
\overrightarrow{\mathrm{dP}} \cdot \vec{p}=w+\vec{\Omega} \cdot(\overrightarrow{O P} \wedge \vec{p})
$$

L'élimination de $u, v, w$ de ces relations donne la relation suivante :

$$
\begin{aligned}
& \mathrm{d}(F, \vec{f})= k t \overrightarrow{\mathrm{dT}} \cdot \vec{t}+k s \cdot \overrightarrow{\mathrm{d} S} \cdot \vec{s}+k p \cdot \overrightarrow{\mathrm{dP}} \cdot \vec{p} \\
&+\vec{\Omega}[(\overrightarrow{O F} \wedge \vec{f})-(k t \cdot \overrightarrow{O T} \wedge \vec{t}) \\
&\quad-(k s \cdot \overrightarrow{O S} \wedge \vec{s})-(k p \cdot \overrightarrow{O P} \wedge \vec{p})]
\end{aligned}
$$

avec

$$
\begin{aligned}
& k t=\frac{f x}{t x} \\
& k s=\frac{1}{s y} \cdot\left(f y-\frac{f x \cdot t y}{t x}\right) \\
& k p=\left(f z-\frac{f x \cdot t z}{t x}-\frac{s z}{s y}\left(f y-\frac{f x \cdot t y}{t x}\right)\right)
\end{aligned}
$$

Il faut trouver les points $S$ et $P$ tels que cette relation soit vraie quel que soit $\vec{\Omega}$, ce qui impose d'annuler le crochet :

$$
\begin{aligned}
X p & =[-(Z f . f x-X f . f z)+K t(Z t . t x-X t . t z) \\
& -K s . X s . s z] / K p \\
Y p & =[(Y f . f z-Z f . f y)-K t(Y t . t z-Z t . t y) \\
& -K s .(Y s . s z-Z s . s y)] / K p \\
X s & =[(X f . f y-Y f . f x)-K t(X t . t y-Y t . t x)] / K s . s y
\end{aligned}
$$

avec $S 1(X 1, Y 1, Z 1)$ et $S 2(X 2, Y 2, Z 2)$

$$
\begin{aligned}
& Y s=Y 1+(Y 2-Y 1) \cdot(X s-X 1) /(X 2-X 1) \\
& Z s=Z 1+(Z 2-Z 1) \cdot(X s-X 1) /(X 2-X 1)
\end{aligned}
$$

Les points $P, S$ et $T$ sont appelés les points d'analyse. Ils appartiennent à la pièce terminale. $P$ est sur le plan de contact primaire. $S$ appartient à la ligne de contact secondaire, $T$ est le point de contact tertiaire. Comme ces points sont sur les surfaces de contact, leur déplacement dépend directement des écarts des points d'appui.

Pour déterminer le déplacement maximal du point $F$, il suffit donc de déterminer le déplacement maximum des points $P, S$ et $T$ définis ci-dessus, à l'aide de la relation suivante :

$$
\mathrm{d}(F, \vec{f})=k p \cdot \mathrm{d}(P, \vec{p})+k s \cdot \mathrm{d}(S, \vec{s})+k t \cdot \mathrm{d}(T, \vec{t})
$$

\subsection{Cas particuliers}

Si $k p, k s$ ou $k t=0$, la formule générale n'est plus valable. Quelques cas particuliers sont donnés ci-dessous :

- Si $k t=k s=0(f z= \pm 1)$ : La droite d'analyse est perpendiculaire au plan primaire

$$
\mathrm{d}(F, \vec{f})=\mathrm{d}(P, \vec{p}) \text { avec } P x=F x \text { et } P y=F y
$$

- Si $k t=0, k s \# 0, k p \# 0:$ La tertiaire n'intervient pas :

Sur $x: F y . f z-F z . f y-k s . S y . s z+k s . S z . s y-k p . P y=0$

Sur $y: F z . f x-F x . f z+k s . S x . s z+k p . P x=0$

Sur $z: F x . f y-F y . f x-k s . S x . s y=0$

La dernière relation donne $S x$. Suivant l'arête haute ou basse, on obtient $S y$ et $S z$, ce qui permet de calculer $P x$ et $P y$. 


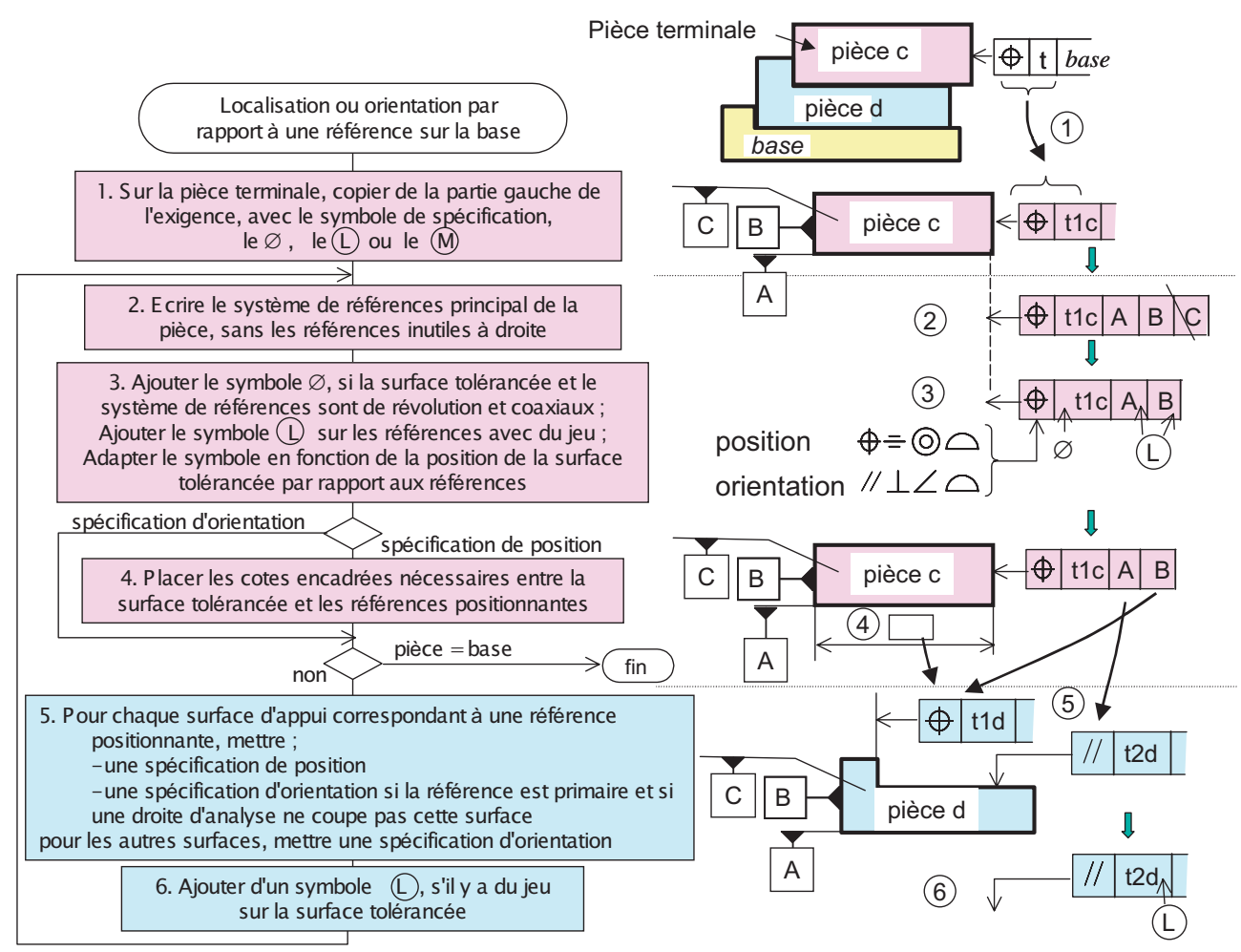

Fig. 11. Organigramme de la méthode CLIC.

- Si $k s=0$ et $k p \# 0$ : Le point $S x$ est rejeté à l'infini. Seul le parallélisme intervient. En fait, il n'est pas possible d'annuler la composante suivant $z$ de l'effet de la rotation. On obtient :

$$
\begin{aligned}
& P x=[-F z . f x-F x \cdot f z+k t . T z \cdot t x-k t \cdot T x \cdot t z] / k p \\
& P y=[F y \cdot f z-F z \cdot f y-k t . T y \cdot t z+k t \cdot T z \cdot t y] / k p \\
& m z=F x \cdot f y-F y \cdot f x-k t \cdot T x \cdot t y+k t \cdot T y \cdot t x \\
& \mathrm{~d}(F, f)=k t \cdot \mathrm{d}(T, \vec{t})+k p \cdot \mathrm{d}(P, \vec{p})+m z \cdot a(\Delta, \overrightarrow{\varepsilon z}) \\
& \quad(\varepsilon=+1 \quad \text { si } m z>0, \varepsilon=-1 \quad \text { si } m z<0)
\end{aligned}
$$

$\Delta$ est une droite de la face secondaire qui est parallèle au plan primaire. $a(\Delta, \overrightarrow{\varepsilon z})$ donne l'angle de rotation maxi de ce plan.

Les autres cas particuliers sont traités de manière similaire.

\subsection{Cotation des pièces influentes}

Dans le cas d'une liaison surfacique, le déplacement des points $P, S$, et $T$ dépendent des tolérances qui sont fixées par la cotation. La méthode CLIC est basée sur l'algorithme itératif de la figure 11 [6]. Les spécifications obtenues sont principalement des localisations et des orientations des surfaces d'appui par rapport au système de références principal de la pièce. Cet algorithme analyse globalement l'ensemble de la surface terminale, et pas uniquement chaque point de discrétisation, ce qui donne une seule cotation pour chaque surface.

\subsection{Influence des défauts des appuis dans une jonction plan/plan}

Dans le cas d'une jonction surfacique orthomorphe plan/plan (Fig. 12), la relation est très simple :

$$
\mathrm{d}(F 1, \vec{f})=\mathrm{d}(P 1, \vec{p}) \cdot \sin \varphi+\mathrm{d}(S 1, \vec{s}) \cdot \cos \varphi
$$

Le point primaire $P 1$ obtenu numériquement par les formules en 4.2 , est facilement identifiable graphiquement. Les deux droites passant par $S 1$ et par $P 1$ se coupent en $I 1$, qui est sur la droite d'analyse passant par F1 (Fig. 12a). On aura donc une nouvelle droite d'analyse primaire passant par $P 1$ de normale $\vec{p}$ et une droite d'analyse secondaire passant par $S 1$, de normale $\vec{s}$. Chaque point fonctionnel $F 1, F 2 \ldots$ donnera donc des droites d'analyse différentes.

Le problème est donc de déterminer la ligne de contact secondaire qui peut être l'arête supérieure ou l'arête inférieure commune aux deux faces. La figure $12 \mathrm{~b}$ montre que, le déplacement de $P$ est maximum lorsque la face d'appui s'incline, induisant un contact secondaire sur l'arête supérieure.

La cotation imposée par la méthode CLIC est donnée figure $13 \mathrm{a}$ et $13 \mathrm{~b}$. Le défaut maxi de la surface terminale $(t 1 a / 2)$ s'ajoute au déplacement du point $F$ dû aux déplacements des appuis :

$$
\mathrm{d}(F, \vec{f})=t 1 a / 2+\mathrm{d}(P, \vec{p}) \cdot \sin \varphi+\mathrm{d}(S, \vec{s}) \cdot \cos \varphi
$$

Le déplacement du point $P$ est limité par les tolérances de la face primaire (Fig. 13c) :

$$
\mathrm{d}(P, \vec{p})=t 1 b / 2+t 2 b \times L b / E b
$$




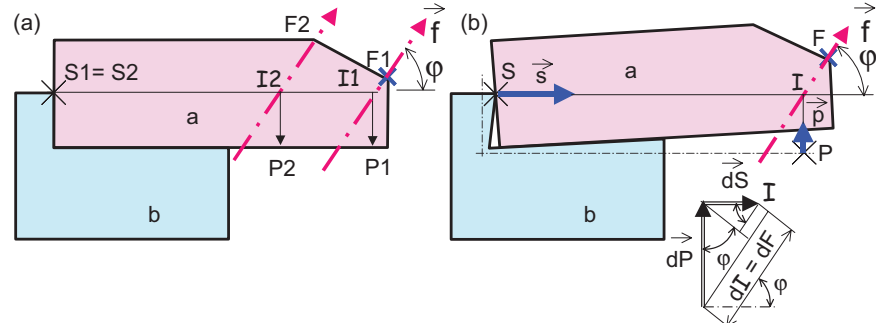

Fig. 12. Détermination des points d'analyse.
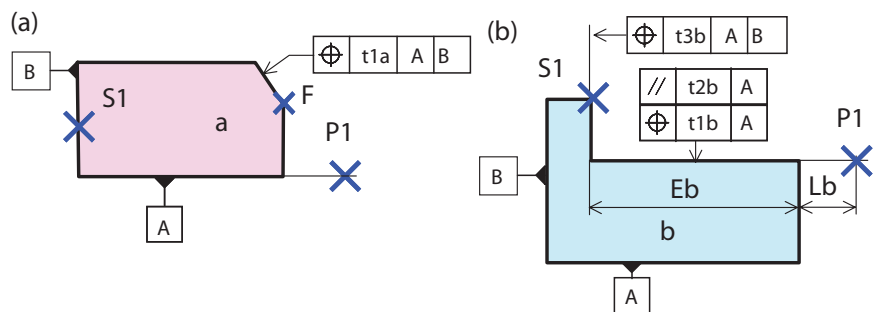

(c) Tolérances

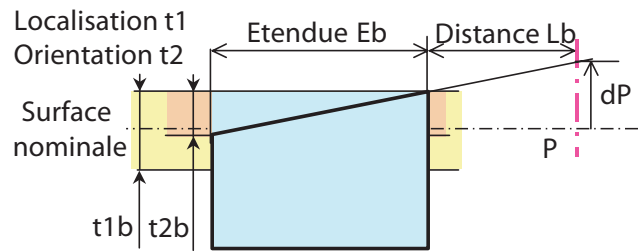

Fig. 13. Influence des tolérances.
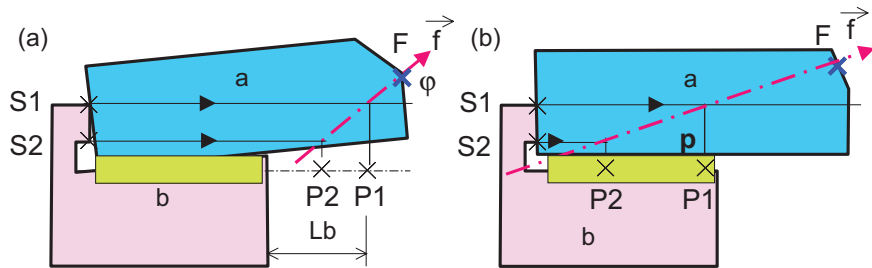

Fig. 14. Cas simples de transfert.

Le déplacement du point $S$ est limité par la tolérance de la face secondaire : $\mathrm{d}(S, \vec{s})=t 3 b / 2$

D'où la relation complète :

$$
\mathrm{d}(F, \vec{f})=t 1 a / 2+t 1 b / 2+t 2 b \times L b / E b+t 3 b / 2
$$

La figure 14a montre qu'a priori, il y a deux arêtes $S 1$ et $S 2$ de contact possible.

Cependant, $\mathrm{d}(S 1, \vec{s})=\mathrm{d}(S 2, \vec{s})$ et $\mathrm{d}(P 1, \vec{p})>$ $\mathrm{d}(P 2, \vec{p})$. On peut donc éviter d'étudier le cas $S 2, P 2$ car on ne cherche que la configuration qui donne le déplacement maximal de $F$.

La figure $14 \mathrm{~b}$ montre un cas où les points $P 1$ et $P 2$ sont sur la face. On a alors simplement $\mathrm{d}(P, \vec{p})=t 1 b / 2$. La méthode CLIC prend en compte cette particularité et n'impose pas de spécification d'orientation $t 2 b$ (règle de l'étape 5 de la Fig. 11).

La figure 15 montre une configuration particulière. Si la pente $\alpha$ est positive, le contact a lieu sur l'arête supérieure en $S 1$, avec un point d'analyse $P 1$. Si $\alpha$ est négatif, le contact a lieu sur l'arête inférieure en $S 2$ et le

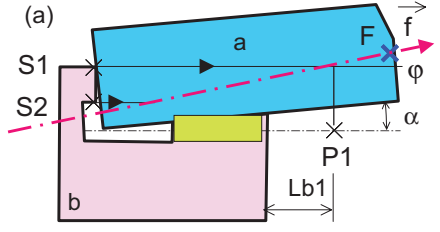

$\mathrm{dP} 1=[\mathrm{t} 1 \mathrm{~b} / 2+\mathrm{t} 2 \mathrm{~b} \cdot \mathrm{Lb} 1 / \mathrm{Eb}]$

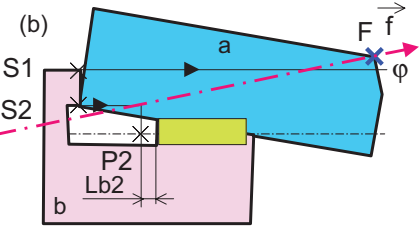

$\mathrm{dP} 2=[\mathrm{t} 1 \mathrm{~b} / 2+\mathrm{t} 2 \mathrm{~b} \cdot \mathrm{Lb} 2 / E \mathrm{~b}]$
Fig. 15. Comparaison des deux maximums en fonction de $\alpha$.
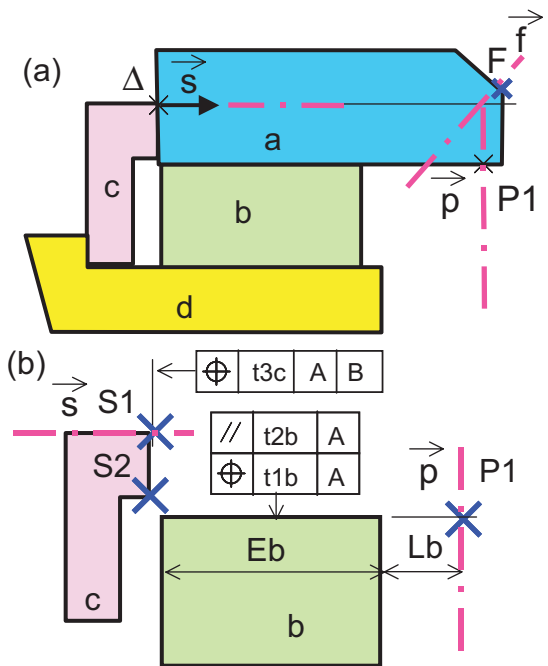

Fig. 16. Transfert dans des branches parallèles.

point d'analyse primaire est $P 2$. Il faut donc retenir le cas avec le plus grand $L b$.

\subsection{Influence des défauts dans une jonction parallèle}

Un montage série comme celui de la figure 9 est étudié en cumulant directement l'influence de chaque jonction sur la surface terminale. Lorsque les pièces d'appui d'une jonction sont différentes, les points $P, S$ et $T$ appartiennent à des pièces différentes (Fig. 16). Il faut alors étudier respectivement les déplacements de $P$, de $S$ et de $T$, dans chaque sous-branche primaire, secondaire et tertiaire séparément en considérant les droites d'analyse correspondante.

À partir de la droite d'analyse $(F, \vec{f})$, cette méthode donne les déplacements : $\mathrm{d}(F, \vec{f})=t 1 a / 2+$ $\mathrm{d}(P, \vec{p}) \cdot \sin \varphi+\mathrm{d}(S, \vec{s}) \cdot \cos \varphi$ et les droites d'analyse $(P 1, \vec{p})$ et $(S 1, \vec{s})$.

Chaque branche parallèle est ensuite étudiée séparément en considérant les points $S 1$ et $P 1$ comme les points des surfaces terminales à étudier. Comme il n'est pas possible a priori de savoir quel est le point $S 1$ ou $S 2$ qui se déplace le plus, il faut établir une légère combinatoire pour déterminer le déplacement maximum.

En synthèse, lorsqu'il n'y a qu'une pièce d'appui, on étudie toujours le déplacement du point de la surface terminale avec une seule droite d'analyse. Lorsque les appuis sont sur des pièces différentes, il faut étudier chaque branche avec une droite d'analyse spécifique. Par contre, 


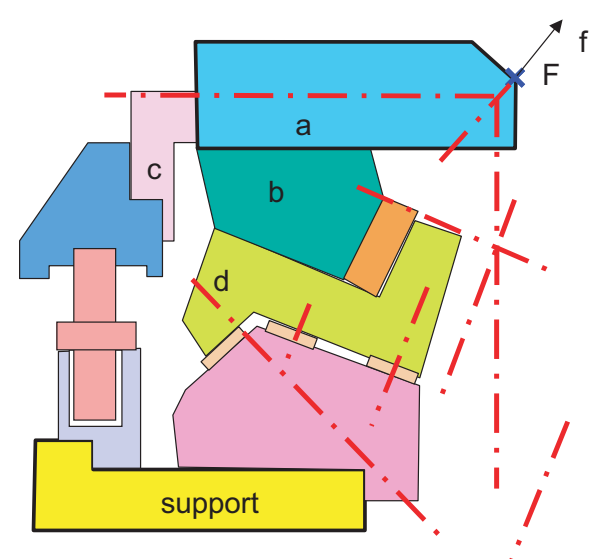

Fig. 17. Changements de direction d'analyse.

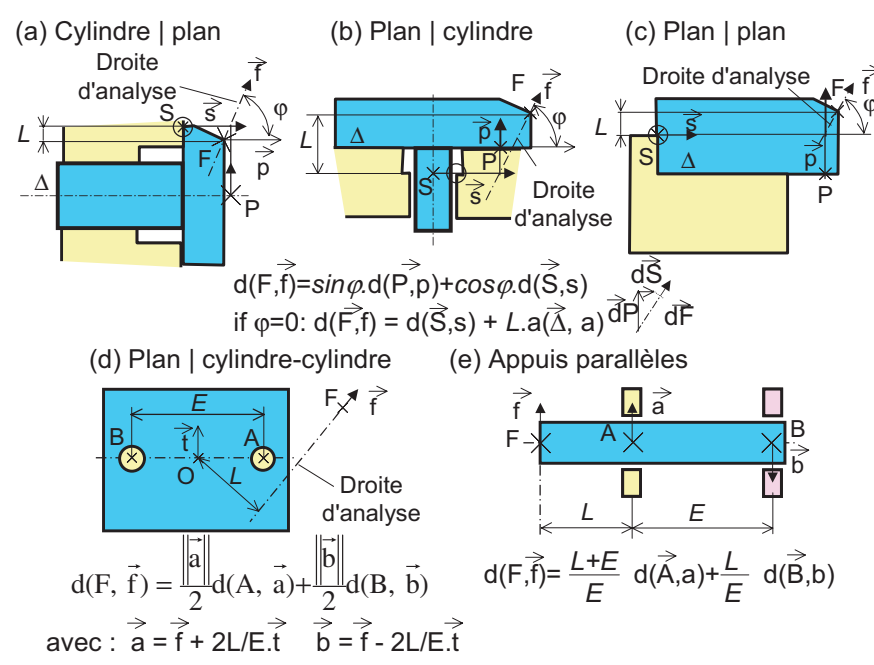

Fig. 18. Relations classiques de transfert.

lorsque les branches convergent dans une même pièce, il est possible de reprendre la droite d'analyse utilisée avant la séparation, ce qui évite la multiplication des combinaisons. La figure 17 montre l'application à un mécanisme générique de cette méthode dite « des droites d'analyse ».

\subsection{Autres jonctions}

La figure 18 donne les formules de transfert pour les jonctions classiques.

\section{Décomposition d'une exigence complexe}

\subsection{Règle de décomposition en sous-exigences}

L'algorithme de la figure 11 et la méthode de transfert conviennent pour une exigence de position d'une surface terminale par rapport à la base du mécanisme. Toutefois, la figure 1 montre qu'il existe une grande diversité d'exigences à traiter : des distances entre deux surfaces, des spécifications en zone commune ou encore sur des

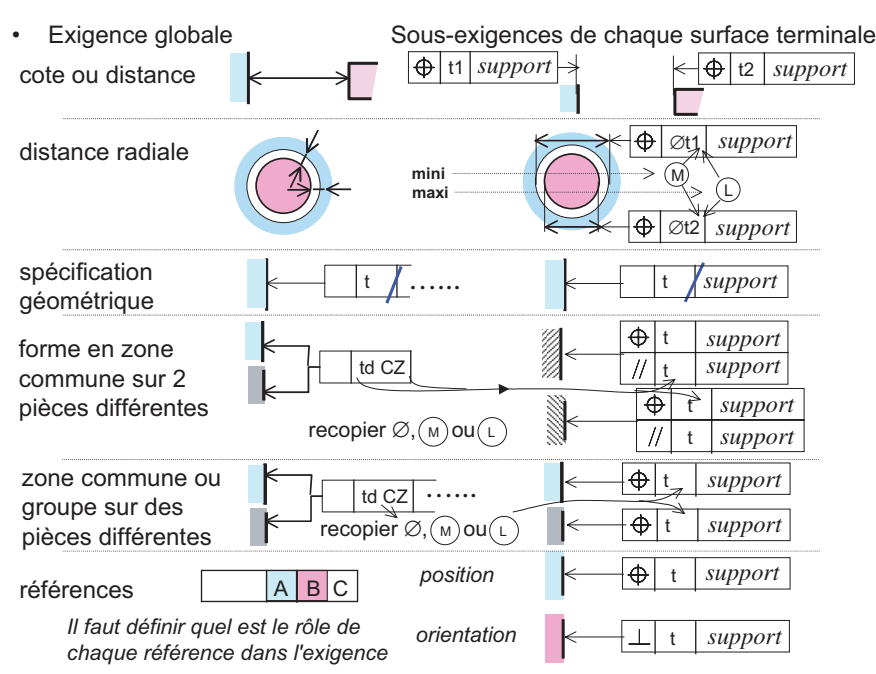

Fig. 19. Décomposition d'une exigence complexe en sousexigences.

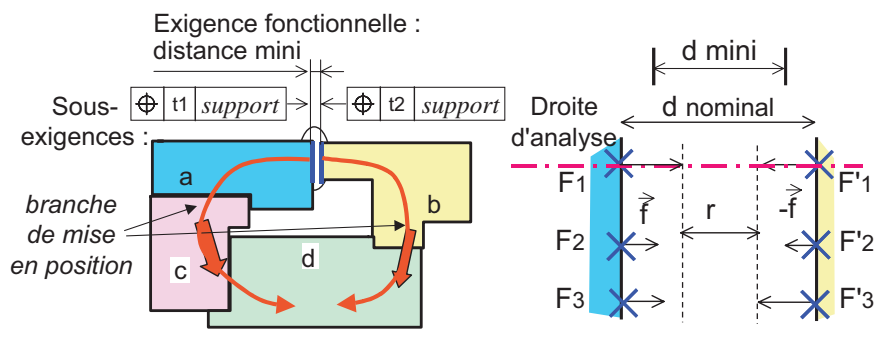

Fig. 20. Décomposition d'une distance.

spécifications de position ou d'orientation par rapport à un système de références. Le transfert devient complexe lorsque les surfaces terminales de ces exigences n'appartiennent pas à la même pièce.

Lorsqu'une exigence relie plusieurs surfaces fonctionnelles appartenant à des pièces différentes, la méthode CLIC impose de décomposer cette exigence en plusieurs sous-exigences à l'aide des règles synthétisées figure 19.

\subsection{Exigence de distance}

Une exigence de distance est transformée en deux sous-exigences de localisation (Fig. 20), ce qui permet d'utiliser deux fois la méthode pour les liaisons série vue 4. La résultante mini correspondant à l'exigence dépend des déplacements maxi de chaque surface terminale respectivement étudiée dans les directions $\vec{f}$ et $-\vec{f}$.

\subsection{Exigence de rectitude en zone commune}

La figure 21a montre une exigence de rectitude en zone commune qui est décomposée en sous-exigences d'orientation et de position (Fig. 21b). Une simple analyse de la définition de la zone de rectitude permet d'établir la démarche suivante. 


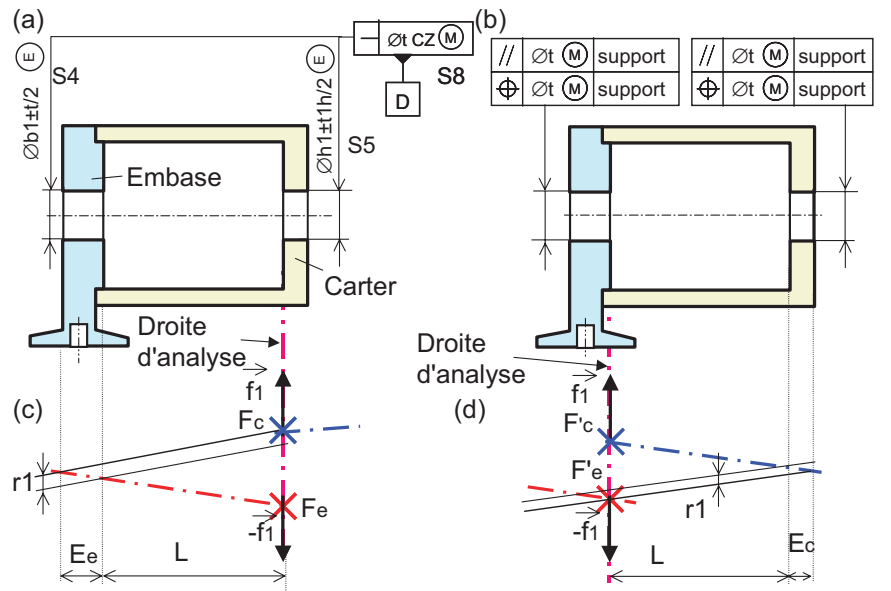

Fig. 21. Relations pour une rectitude en zone commune.

Pour chaque direction de discrétisation $\vec{f} i$, il faut étudier deux cas :

En (c), la droite d'analyse est à l'extrémité gauche de l'alésage du carter. $F_{\mathrm{c}}$ est l'extrémité gauche de l'axe de l'alésage du carter. $F_{\mathrm{e}}$ est un point de l'axe de l'embase dans le même plan.

En (d), la droite d'analyse est à l'extrémité droite de l'alésage de l'embase. $F_{\mathrm{e}}^{\prime}$ est l'extrémité droite de l'axe de l'alésage de l'embase. $F_{\mathrm{c}}^{\prime}$ est un point de l'axe du carter dans le même plan.

La relation de décomposition de la rectitude est donc la suivante :

$$
\begin{aligned}
& r 1=\max \left\{\frac{E_{e}}{E_{e}+L}\left[\mathrm{~d}\left(F_{\mathrm{c}}, \vec{f}\right)+\mathrm{d}\left(F_{\mathrm{e}},-\vec{f}\right)\right]\right. \\
&\left.\frac{E_{\mathrm{c}}}{E_{\mathrm{c}}+L}\left[\mathrm{~d}\left(F_{\mathrm{c}}^{\prime}, \vec{f}\right)+\mathrm{d}\left(F_{\mathrm{c}}^{\prime},-\vec{f}\right)\right]\right\}
\end{aligned}
$$

\subsection{Exigence de position par rapport à un système de références}

La figure 22 donne le principe de décomposition d'un système de références. L'exigence est définie par rapport aux surfaces $A$ et $B$ qui ne sont pas sur la même pièce. La surface nominale est définie par des cotes et des angles encadrés. Dans la méthode CLIC [2], les branches de mise en position de la surface fonctionnelle et des surfaces de références $\mathrm{A}$ et $\mathrm{B}$ convergent dans le support. Le repère $\mathrm{CAO}$ du support est donc considéré comme un repère fixe.

Il s'agit de déterminer les déplacements des trois surfaces, ce qui permet de construire le système de références $\mathrm{AB}$ et la position de la surface nominale après ce déplacement. Le plan de référence primaire est tangent à la surface $\mathrm{A}$. Le plan de référence secondaire est perpendiculaire à $\mathrm{A}$ et tangent à la surface $\mathrm{B}$. Il faut donc considérer les différents cas de contacts secondaires (Fig. 23). À partir de la droite d'analyse $(F, \vec{f})$, il y a deux cas de contact secondaire $(S 1, \vec{s})$ et $(S 2, \vec{s})$ ce qui donne respectivement les deux cas primaires $(P 1, \vec{p})$ et $(P 2, \vec{p})$.

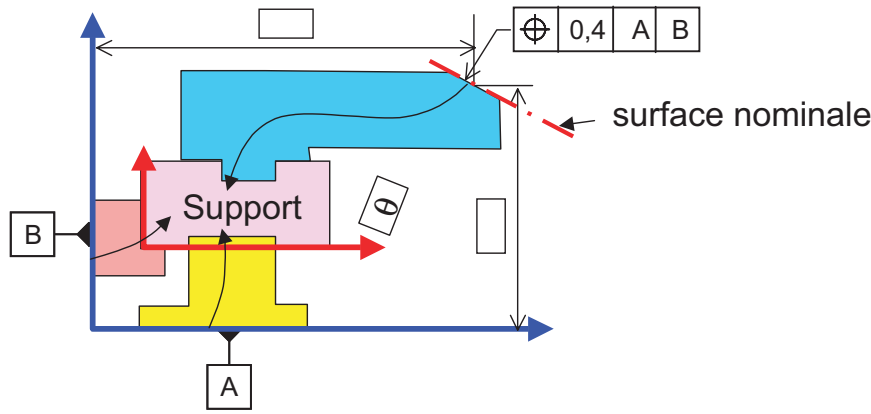

Fig. 22. Localisation par rapport à un système de références.

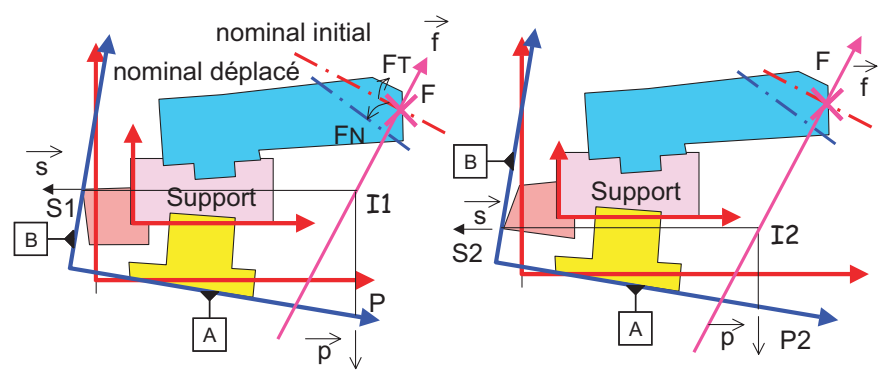

Fig. 23. Deux cas de contact secondaire.

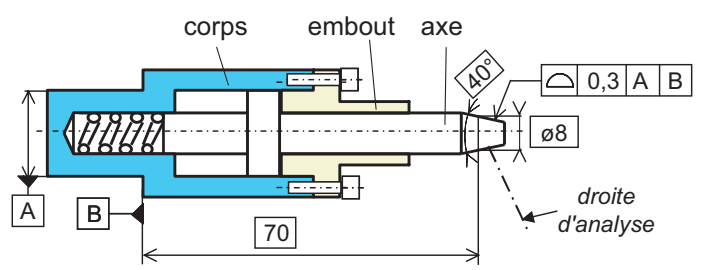

Fig. 24. Exigence de position d'une surface conique.

L'écart résultant est alors la somme des trois déplacements :

$$
\begin{aligned}
& r=d(\overrightarrow{F, f)}+d(P, \vec{p}) \cdot \sin \varphi+d(\vec{S}, \vec{s}) \cdot \cos \varphi \\
& \begin{array}{cll}
\text { surface } & \text { surface } & \text { surface } \\
\text { fonctionnelle } & \text { primaire } & \text { secondaire }
\end{array}
\end{aligned}
$$

Chaque déplacement est calculé par rapport au support par la méthode générale.

\section{Application}

\subsection{Exigence étudiée}

L'exigence étudiée est une exigence de position de la surface conique par rapport au système de références de la base (Fig. 24).

La figure 25 schématise les différents défauts qui déplacent les points du cône.

Pour cette exigence, l'algorithme figure 11 génère la cotation de la figure 26 qui est fortement basée sur les états virtuels au minimum de matière [7]. 


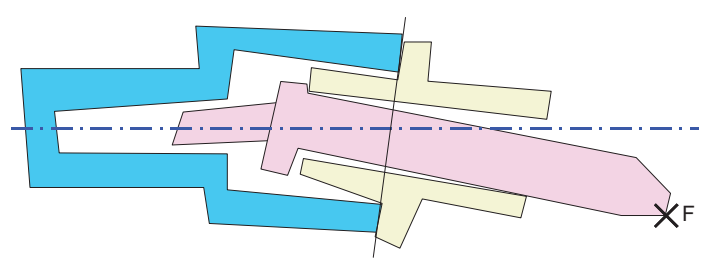

Fig. 25. Cumul 3D des défauts.

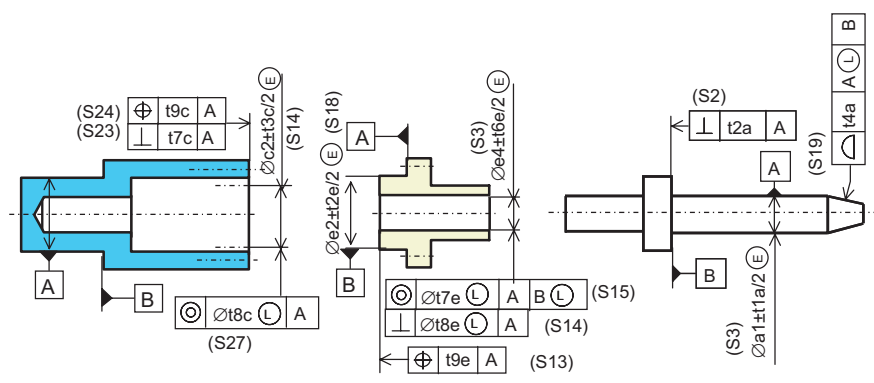

Fig. 26. Cotation des pièces. (a)

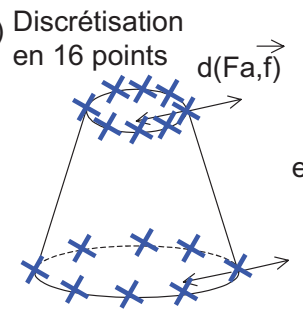

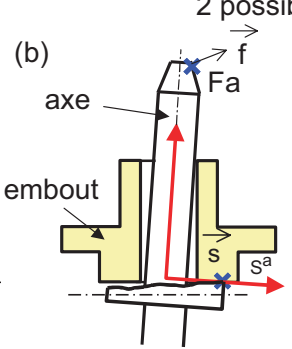

2 possibilités de contact
Fig. 27. Analyse des configurations.

\subsection{Calcul 3D de la résultante}

La figure 27 montre la discrétisation du cône en 16 points, avec des normales perpendiculaires à la surface du cône. Le calcul commence par l'étude de jonction axe/embout. Si le défaut de perpendicularité de l'épaulement est plus important que l'angle maxi permis par le jeu dans l'alésage, le contact a lieu en $S^{\mathrm{a}}$, du côté de $\vec{f}$ (Fig. 27b), si le défaut de perpendicularité est plus faible, le contact a lieu en $S^{\prime a}$, du côté opposé à $\vec{f}$ (Fig. 27c).

Une procédure VBA génère pour les 16 points fonctionnels, les deux relations correspondant aux deux contacts en $S$ et $S^{\prime}$. Le déplacement maxi d'un point $F$ est le plus petit ( $\mathrm{min}$ ) des déplacements avec les contacts en $S$ et $S^{\prime}$. Le déplacement maxi de tous les points sera le plus grand des 16 déplacements.

La figure 28a montre la droite d'analyse passant par $F^{\text {a }}$, le point secondaire $S^{\text {a }}$ de l'axe sur le bord de l'épaulement et le point primaire $P^{\mathrm{a}}$. Le système de références de l'axe passe par $S^{\mathrm{a}}$. La figure $28 \mathrm{~b}$ montre l'étape suivante obtenue en «soudant » l'axe et l'embout pour étudier l'influence de la jonction embout/corps sur le déplacement du point $F$. Le contact secondaire a lieu en $S^{e}$, sur le bord de la portée cylindrique. On en déduit la position du point d'analyse primaire $P_{\mathrm{e}}$.

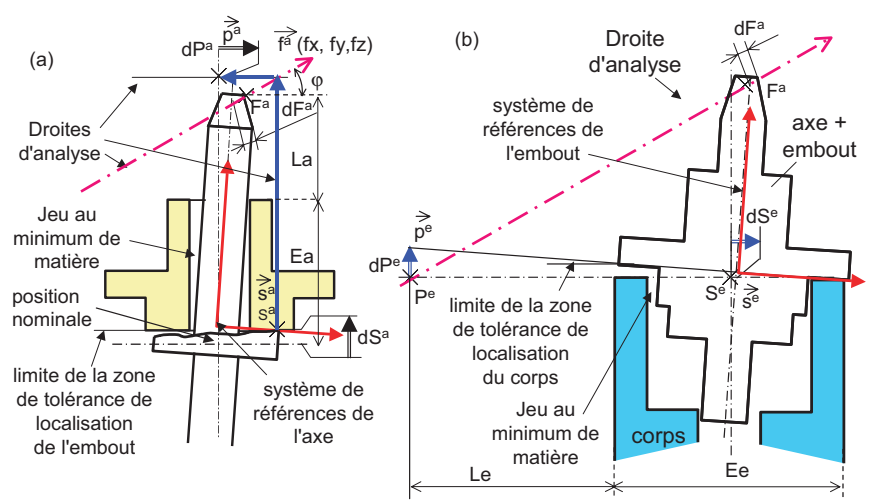

Fig. 28. Influence des deux jonctions.

\begin{tabular}{|c|c|c|c|}
\hline & & & \\
\hline & & $\min$ & $.93^{*} \mathrm{~d} 7 \mathrm{a}(42)+.34^{*} \mathrm{~d} 3 \mathrm{e}(82)+.93^{*} \mathrm{~d} 8 \mathrm{e}(83)+.34^{*} \mathrm{~d} 3 \mathrm{a}(43)+1 * 8 \mathrm{*}(2)$ \\
\hline & 2 & & $.93 * \mathrm{~d} 7 \mathrm{a}(44)+.34 * \mathrm{~d} 3 \mathrm{e}(82)+.93^{*} \mathrm{~d} 8 \mathrm{e}(83)+.34 * \mathrm{~d} 3 \mathrm{a}(45)+.34 * \mathrm{p} 3 \mathrm{a}+1^{*} \mathrm{t} 8 \mathrm{a}(2)$ \\
\hline & & $\min$ & $.93^{*} \mathrm{~d} 7 \mathrm{a}(46)+.34^{*} \mathrm{~d} 3 \mathrm{e}(84)+.93 * \mathrm{~d} 8 \mathrm{e}(85)+.34^{*} \mathrm{~d} 3 \mathrm{a}(47)+1 * 8 \mathrm{a}(3)$ \\
\hline & 3 & & $.93^{*} \mathrm{~d} 7 \mathrm{a}(48)+.34^{*} \mathrm{~d} 3 \mathrm{e}(84)+.93^{*} \mathrm{~d} 8 \mathrm{e}(85)+.34^{*} \mathrm{~d} 3 \mathrm{a}(49)+.34^{*} \mathrm{p} 3 \mathrm{a}+1^{*} \mathrm{z} 8 \mathrm{a}(3)$ \\
\hline 5 & & $\min$ & $.93^{*} \mathrm{~d} 7 \mathrm{a}(50)+.34^{*} \mathrm{~d} 3 \mathrm{e}(86)+.93^{*} \mathrm{~d} 8 \mathrm{e}(87)+.34^{*} \mathrm{~d} 3 \mathrm{a}(51)+1 * \mathrm{r} \mathrm{a}(4)$ \\
\hline 6 & 4 & & $.93 * \mathrm{~d} 7 \mathrm{a}(52)+.34 * \mathrm{~d} 3 \mathrm{e}(86)+.93 * \mathrm{~d} d 8 \mathrm{e}(87)+.34 * \mathrm{~d} 3 \mathrm{a}(53)+.34 * \mathrm{p} 3 \mathrm{a}+1 * \mathrm{t} 8 \mathrm{a}(4)$ \\
\hline & & $\min$ & $.93^{*} \mathrm{~d} 7 \mathrm{a}(54)+.34^{*} \mathrm{~d} 3 \mathrm{e}(88)+.93 * \mathrm{~d} 8 \mathrm{e}(89)+.34 * \mathrm{~d} 3 \mathrm{a}(55)+1 * \mathrm{ag}(5)$ \\
\hline & 5 & & $.93^{*} \mathrm{~d} 7 \mathrm{a}(56)+.34^{*} \mathrm{~d} 3 \mathrm{e}(88)+.93^{*} \mathrm{~d} 8 \mathrm{e}(89)+.34 * \mathrm{~d} 3 \mathrm{a}(57)+.34^{*} \mathrm{p} 3 \mathrm{a}+1^{*} \mathrm{t} 8 \mathrm{a}(5)$ \\
\hline & & $\min$ & $.93 * \mathrm{~d} 7 \mathrm{a}(58)+.34 * \mathrm{~d} 3 \mathrm{e}(90)+.93 * \mathrm{~d} 8 \mathrm{e}(91)+34 * \mathrm{~d} 3 \mathrm{a}(45)+1 * \mathrm{t} 8 \mathrm{a}(6)$ \\
\hline 10 & 6 & & $.93 * \mathrm{~d} 7 \mathrm{a}(59)+.34 * \mathrm{~d} 3 \mathrm{e}(90)+.93 * \mathrm{~d} 8 \mathrm{e}(91)+.34 * \mathrm{~d} 3 \mathrm{a}(43)+.34 * \mathrm{p} 3 \mathrm{a}+1 * \mathrm{t} 8 \mathrm{a}(6)$ \\
\hline 11 & & $\min$ & $.93 * d 7 a(60)+.34 * d 3 e(92)+.93 * d 8 \mathrm{e}(93)+.34 * \mathrm{~d} 3 \mathrm{a}(49)+1 * \mathrm{*} 8 \mathrm{a}(7)$ \\
\hline 12 & 7 & & $.93 * \mathrm{~d} 7 \mathrm{a}(61)+.34^{*} \mathrm{~d} 3 \mathrm{e}(92)+.93 * \mathrm{~d} d 8 \mathrm{e}(93)+.34 * \mathrm{~d} 3 \mathrm{a}(47)+.34^{*} \mathrm{p} 3 \mathrm{a}+1 * \mathrm{t} 8 \mathrm{a}(7)$ \\
\hline 13 & & $\min$ & $.93^{*} \mathrm{~d} 7 \mathrm{a}(62)+.34^{*} \mathrm{~d} 3 \mathrm{e}(94)+.93 * \mathrm{~d} d 8 \mathrm{e}(95)+.34 * \mathrm{~d} 3 \mathrm{a}(53)+1 *+8 \mathrm{a}(8)$ \\
\hline 14 & 8 & & $.93^{*} \mathrm{~d} 7 \mathrm{a}(63)+.34^{*} \mathrm{~d} 3 \mathrm{e}(94)+.93^{*} \mathrm{~d} 8 \mathrm{e}(95)+.34^{*} \mathrm{~d} 3 \mathrm{a}(51)+.34^{*} \mathrm{p} 3 \mathrm{a}+1^{*}+8 \mathrm{a}(8)$ \\
\hline 15 & & $\min$ & $.93 * \mathrm{~d} 7 \mathrm{a}(64)+.34 * \mathrm{~d} 3 \mathrm{e}(96)+.93^{*} \mathrm{~d} 8 \mathrm{e}(97)+.34^{*} \mathrm{~d} 3 \mathrm{a}(57)+1 * \mathrm{t} 8 \mathrm{a}(9)$ \\
\hline 16 & 9 & & $.93 * d 7 a(65)+.34^{*} d 3 e(96)+.93 * d 8 e(97)+34^{*} d 3 a(55)+34^{*}$ p3a $+1 *+8 a(9)$ \\
\hline 17 & 10 & $\min$ & $93 * \mathrm{~d} 7 \mathrm{a}(66)+.34 * \mathrm{~d} 3 \mathrm{e}(98)+.93 * \mathrm{~d} 8 \mathrm{e}(83)+.34 * \mathrm{~d} 3 \mathrm{a}(43)+1 * \mathrm{t} 8 \mathrm{a}(10)$ \\
\hline 18 & 10 & & $93^{*} \mathrm{~d} 7 \mathrm{a}(67)+.34^{*} \mathrm{~d} 3 \mathrm{e}(98)+.93^{*} \mathrm{~d} 8 \mathrm{e}(83)+.34^{*} \mathrm{~d} 3 \mathrm{a}(45)+.34^{*} \mathrm{p} 3 \mathrm{a}+1^{*} \mathrm{t} 8 \mathrm{a}(10)$ \\
\hline 19 & & & $93^{*} \mathrm{~d} 7 \mathrm{a}(68)+.34^{*} \mathrm{~d} 3 \mathrm{e}(99)+.93^{*} \mathrm{~d} 8 \mathrm{e}(85)+.34^{*} \mathrm{~d} 3 \mathrm{a}(47)+1 * \mathrm{ra}(11)$ \\
\hline 20 & 11 & & $93 * \mathrm{~d} 7 \mathrm{a}(69)+.34 * \mathrm{~d} 3 \mathrm{e}(99)+.93 * \mathrm{~d} d 8 \mathrm{e}(85)+.34 * \mathrm{~d} 3 \mathrm{a}(49)+.34^{*} \mathrm{p} 3 \mathrm{a}+1^{*} \mathrm{t} 8 \mathrm{a}(11)$ \\
\hline 21 & & $\min$ & $93^{*} \mathrm{~d} 7 \mathrm{a}(70)+.34^{*} \mathrm{~d} 3 \mathrm{e}(100)+.93^{*} \mathrm{~d} 8 \mathrm{e}(87)+.34^{*} \mathrm{~d} 3 \mathrm{a}(51)+1^{*} \mathrm{t} 8 \mathrm{a}(12)$ \\
\hline 22 & 12 & & $93^{*} \mathrm{~d} 7 \mathrm{a}(71)+.34^{*} \mathrm{~d} 3 \mathrm{e}(100)+.93 * \mathrm{~d} 8 \mathrm{e}(87)+.34^{*} \mathrm{~d} 3 \mathrm{a}(53)+.34^{*} \mathrm{p} 3 \mathrm{a}+1^{*} \mathrm{t} 8 \mathrm{a}(12)$ \\
\hline 23 & & & $93 * \mathrm{~d} 7 \mathrm{a}(72)+.34^{*} \mathrm{~d} 3 \mathrm{e}(101)+.93 * \mathrm{~d} 8 \mathrm{e}(89)+.34^{*} \mathrm{~d} 3 \mathrm{a}(55)+1^{*} \mathrm{t} 8 \mathrm{a}(13)$ \\
\hline 24 & 13 & & $93^{*} \mathrm{~d} 7 \mathrm{a}(73)+.34^{*} \mathrm{~d} 3 \mathrm{e}(101)+.93^{*} \mathrm{~d} 8 \mathrm{e}(89)+.34^{*} \mathrm{~d} 3 \mathrm{a}(57)+.34^{*} \mathrm{p} 3 \mathrm{a}+1^{*} \mathrm{t} 8 \mathrm{a}(13)$ \\
\hline 25 & 14 & $\min$ & $93 * d 7 \mathrm{a}(74)+.34 * \mathrm{~d} 3 \mathrm{e}(102)+.93 * \mathrm{~d} d 8 \mathrm{e}(91)+.34 * \mathrm{~d} 3 \mathrm{a}(45)+1 *+8 \mathrm{a}(14)$ \\
\hline 26 & 14 & & $93^{*} \mathrm{~d} 7 \mathrm{a}(75)+.34^{*} \mathrm{~d} 3 \mathrm{e}(102)+.93 * \mathrm{~d} 8 \mathrm{e}(91)+.34^{*} \mathrm{~d} 3 \mathrm{a}(43)+.34^{*} \mathrm{p} 3 \mathrm{a}+1^{*} \mathrm{t} 8 \mathrm{a}(14)$ \\
\hline 27 & 15 & $\min$ & $93 * \mathrm{~d} 7 \mathrm{a}(76)+.34^{*} \mathrm{~d} 3 \mathrm{e}(103)+.93 * \mathrm{~d} 8 \mathrm{e}(93)+.34^{*} \mathrm{~d} 3 \mathrm{a}(49)+1 * \mathrm{t} 8 \mathrm{a}(15)$ \\
\hline 28 & 15 & & $93^{*} \mathrm{~d} 7 \mathrm{a}(77)+.34^{*} \mathrm{~d} 3 \mathrm{e}(103)+.93 * \mathrm{~d} 8 \mathrm{e}(93)+.34^{*} \mathrm{~d} 3 \mathrm{a}(47)+.34^{*} \mathrm{p} 3 \mathrm{a}+1{ }^{*} \mathrm{t} 8 \mathrm{a}(15)$ \\
\hline 29 & & & $93^{*} \mathrm{~d} 7 \mathrm{a}(78)+.34^{*} \mathrm{~d} 3 \mathrm{e}(104)+.93^{*} \mathrm{~d} 8 \mathrm{e}(95)+.34^{*} \mathrm{~d} 3 \mathrm{a}(53)+1^{*} \mathrm{t} 8 \mathrm{a}(16)$ \\
\hline 30 & 16 & & $93^{*} \mathrm{~d} 7 \mathrm{a}(79)+.34^{*} \mathrm{~d} 3 \mathrm{e}(104)+.93^{*} \mathrm{~d} 8 \mathrm{e}(95)+.34^{*}$ \\
\hline 31 & 17 & $\min$ & $93^{*} \mathrm{~d} 7 \mathrm{a}(80)+.34 * \mathrm{~d} 3 \mathrm{e}(105)+.93 * \mathrm{~d} d 8 \mathrm{e}(97)+.34 * \mathrm{~d} 3 \mathrm{a}(57)+1 * \mathrm{t} 8 \mathrm{a}(17)$ \\
\hline & 17 & & $93^{*} \mathrm{~d} 7 \mathrm{a}(81)+.34^{*} \mathrm{~d} 3 \mathrm{e}(105)+.93^{*} \mathrm{~d} 8 \mathrm{e}(97)+.34^{*} \mathrm{~d} 3 \mathrm{a}(55)+.34^{*} \mathrm{p} 3 \mathrm{a}+1^{*}+8 \mathrm{a}(17)$ \\
\hline
\end{tabular}

Fig. 29. Relations générées pour le calcul des exigences.

\subsection{Calcul 3D de la résultante}

Les relations générées sont récapitulées figure 29. Elles sont sous la forme suivante : $0,93 \times d 7 a(42)$.

0,93 est le coefficient d'influence.

$d 7 a(42)$ est le déplacement de la surface 7 de l'axe (a) au point d'analyse 42 . Le point d'analyse est caractérisé par les coordonnées du point $x, y, z$ et les composantes de la direction d'analyse $f x, f y, f z$.

L'indication t porte sur le déplacement d'un point de la surface terminale. L'indication a porté sur l'angle maxi d'une droite.

L'indication min signifie qu'il faut prendre la valeur mini des deux déplacements du point considéré.

Par exemple, la première relation peut être exprimée à l'aide des points de la figure 28 :

$$
\begin{aligned}
\mathrm{d}\left(F^{\mathrm{a}}, \vec{f}\right)= & 0,93 \cdot \mathrm{d}\left(P^{\mathrm{a}}, \overrightarrow{p a}\right)+0,34 \cdot \mathrm{d}\left(S^{\mathrm{a}}, \overrightarrow{s a}\right) \\
& +0,34 \cdot \mathrm{d}\left(P^{\mathrm{e}}, \overrightarrow{p e}\right)+0,93 \cdot \mathrm{d}\left(S^{e}, \overrightarrow{s e}\right)+t\left(F^{\mathrm{a}}, \vec{f}\right) .
\end{aligned}
$$


Ecart $=\mathrm{t} 4 \mathrm{a} / 2+0,34 \cdot \mathrm{t} 2 \mathrm{a}+$ $0,79 .(\mathrm{e} 4-\mathrm{a} 1+\mathrm{t} 6 \mathrm{e} / 2+\mathrm{t} 1 \mathrm{a} / 2)+$ $0,46 . t 7 e+0,32 . t 8 e+0,17 . t 9 e+$ $0,46 .(c 2-e 2+\mathrm{t} 3 c / 2+\mathrm{t} 2 \mathrm{c} / 2)$ $0,17 \cdot t 9 c+0,83 \cdot t 7 c+0,46 t 8 c$

axe
jeu axe/embout
embout
jeu embout/ corps
corps

Fig. 30. Cumul des influences au pire des cas.

$\left(t\left(F^{\mathrm{a}}, \vec{f}\right)\right.$ est l'écart de la surface terminale).

Ces relations sont ensuite calculées en fonction des tolérances placées sur les pièces, ce qui permet de constituer la feuille de calcul de EXCEL figure 31. Par exemple, la seconde relation de la figure 29 se décline en fonction des tolérances et des dimensions des pièces désignées par la figure 24 sous la forme de la figure 30 .

La figure 31 montre la plage de la feuille de calcul EXCEL correspondant à cette exigence. Les lignes 2 et 3 permettent d'imposer l'exigence, avec notamment les limites de tolérance Haute Qualité et Limite Qualité qui vont permettre la synthèse de tolérances [8]. La partie inférieure est générée automatiquement lors du transfert de cotes. Le visual basic génère les formules de calcul. Les fonctions du tableur assurent ensuite les calculs. Le concepteur peut ainsi vérifier le mode de calcul et, éventuellement, apporter des modifications ou ajouter des termes complémentaires (dilatation, flexion...).

La plage A9 :E15 donne la liste des pièces influentes et les repères des spécifications. La plage F9 :H15 donne le calcul pour le point de contact $S^{\mathrm{a}}$ (Fig. 27b). La plage I9 :K15 donne le calcul pour le point de contact à gauche $S^{\prime a}$ (Fig. 27c), ce qui fait intervenir l'écart de perpendicularité $t 2 a$ de l'axe. Ces deux plages correspondent à un point en haut du cône. Il y a également deux plages voisines similaires pour les points en bas du cône (non représentée). Le calcul a été fait pour 8 points sur chaque cercle, mais, compte tenu de la symétrie de révolution, les relations en fonction des tolérances sont identiques, si bien que seul un exemplaire a été conservé après comparaison automatique des relations.

Chaque plage comporte un calcul au pire des cas, directement basé sur la relation qui a été établie figure 30 (colonnes F et I) et un calcul statistique à côté (développé en Sect. 7).

La cellule F9 calcule la résultante au pire des cas pour le contact en $S a$ et en I9, celui en $S^{\prime} a$. La cellule F7 donne le min de ces deux déplacements.

Les cellules G9 et J9 donnent les résultantes statistiques en fonction du nombre d'écarts types désirés indiqué en G3. G7 est la plus petite de ces valeurs. La cellule F5 indique que la distribution est circulaire et qu'il faut faire le calcul de la résultante par Rayleigh en H5.

La cellule A5 est alors le plus grand de ces déplacements au pire des cas, B5 en statistique. C5 est le plus avantageux de ces deux calculs. La cellule F3 permet de faire le choix pire des cas/statistique pour sélectionner l'écart retenu. L'écart doit donc être inférieur à la moitié de la tolérance. L'indice de qualité en E5 qui est ici inférieur à $100 \%$ montre que l'exigence n'est pas totalement respectée.

\section{Calcul statistique}

\subsection{Principe du calcul}

En 3D, l'écart maxi par rapport au nominal peut dépendre de plusieurs spécifications d'une même pièce et des jeux qui correspondent à l'espace compris entre des pièces. La littérature classique propose une méthode dite quadratique qui consiste à faire la racine carrée de la somme des carrés des tolérances. Cette méthode ne marche pas dans le cas 3D, car les différents écarts ne sont pas indépendants. La méthode CLIC propose de regrouper ce qui est propre à une surface ou à un jeu, pour déterminer l'influence de chaque pièce.

La règle de cumul des tolérances est donnée figure $32[2,9]$. La distribution des écarts dans l'intervalle d'influence d'une pièce est inconnue, car il s'agit d'un calcul prévisionnel. Par défaut, on considère souvent une distribution uniforme (Fig. 32a), avec un coefficient $q=2 \sqrt{3}$. On peut aussi considérer que différents facteurs indépendants interviennent dans cet intervalle et donnent une distribution plus centrée avec $q=4,5$ voire 6 (Fig. 32b). Pour les écarts radiaux dus à des coaxialités, des perpendicularités, on peut adopter une distribution polaire uniforme (Fig. 32c). Pour un jeu bloqué, la distribution est définie par la figure $32 \mathrm{~d}$.

\subsection{Détermination des moyennes et des écarts types}

Pour chaque spécification portée sur un dessin, on a une valeur moyenne, une tolérance et un écart type $\sigma$ calculé en fonction du coefficient $q$. Par exemple, la perpendicularité $t 2 a$ de la face de référence de l'axe est comprise entre 0 et $t 2 a$. La valeur moyenne est donc $t 2 a / 2$, la tolérance $t 2 a$ et l'écart type $\sigma 2 a=t 2 a / 2 \sqrt{3}$.

Le calcul statistique est basé sur les relations de la figure 29. Par exemple, la seconde relation qui donne l'écart maxi est :

$$
\begin{aligned}
& \mathrm{d}\left(F^{\mathrm{a}}, \vec{f}\right)=0,93 \cdot \mathrm{d}\left(P^{\mathrm{a}}, \overrightarrow{p a}\right)+0,34 \cdot \mathrm{d}\left(S^{\mathrm{a}}, \overrightarrow{s a}\right) \\
& +0,34 \cdot \mathrm{d}\left(P^{\mathrm{e}}, \overrightarrow{p e}\right)+0,93 \cdot \mathrm{d}\left(S^{\mathrm{e}}, \overrightarrow{s e}\right)+0,34 \cdot p 3 a+t\left(F^{\mathrm{a}}, \vec{f}\right) .
\end{aligned}
$$

Il faut calculer la moyenne et l'écart type de chaque terme. Les moyennes sont placées dans la plage J9 :J15. Les écarts-types dans la plage K9 :K15.

- $t\left(F^{\mathrm{a}}, \vec{f}\right)$ : écart de la surface terminale. La tolérance est $t 4 a$ : La distribution est supposée uniforme moyenne : 0 , écart type : $\sigma 4 a=t 4 a / 2 \sqrt{3}$, coef. : 1

- $p 3 a$ : interpénétration due à la perpendicularité du plan $3 a$ de l'axe. La distribution supposée uniforme entre 0 et $t 2 a$, moyenne $t 2 a / 2$, écart type : $\sigma 2 a=t 2 a / 2 \sqrt{3}$, coef. : 0,34

$-\mathrm{d}\left(P^{\mathrm{a}}, \overrightarrow{p a}\right)$ : influence du jeu libre axe embout : le calcul, similaire à celui de la figure $13 \mathrm{c}$, donne la relation : $d=J_{\operatorname{maxi}}(0,5+L / E)+t 7 e / 2+t 8 \cdot L / E$ (avec $E=$ longueur de la liaison axe/embout, $L=$ porte-à-faux de $P^{\mathrm{a}}$ d'où $\left.L / E=0,35\right)$. 


\begin{tabular}{|c|c|c|c|c|c|c|c|c|c|c|c|}
\hline & A & B & C & $\mathrm{D}$ & $E$ & $F$ & G & $\mathrm{H}$ & 1 & $\mathrm{~J}$ & $\mathrm{~K}$ \\
\hline \multirow[t]{2}{*}{1} & \multicolumn{11}{|c|}{ E14 position d'une surface quelconque } \\
\hline & Type surface & $\begin{array}{l}\text { Surface } \\
\text { tolérancée }\end{array}$ & $\begin{array}{l}\text { Référence } \\
\text { / Surface + }\end{array}$ & $\begin{array}{l}\text { Haute } \\
\text { Qualité }\end{array}$ & Limite Qualité & $\begin{array}{l}\text { Pre cas } 0 ; \\
\text { Statist } 1 ; \\
\text { Mini } 2\end{array}$ & $\begin{array}{l}\text { Nombre } \\
\text { d'écarts- } \\
\text { types }\end{array}$ & \begin{tabular}{|l|} 
Hiérarchisat \\
ion
\end{tabular} & X théorique & théorique Z & théorique \\
\hline 3 & COP & $\$ a$ & \$1c & 0,2 & 0,4 & 0 & 3 & 3 & 69 & 0 & 0 \\
\hline 4 & $\begin{array}{l}\text { Ecart arithm } \\
\text { résultant }\end{array}$ & \begin{tabular}{|l|} 
Ecart \\
statistique \\
résultant
\end{tabular} & \begin{tabular}{|l} 
Ecart \\
optimal \\
résultant
\end{tabular} & \begin{tabular}{|l} 
Ecart \\
retenu
\end{tabular} & Qualité obtenue & $\begin{array}{l}\text { istribution } \\
1 \text { circulai, } 2 \\
\text { excentr, } 3 \\
\text { ovale }\end{array}$ & $\begin{array}{l}\text { Distribution } \\
\text { excentrée }\end{array}$ & $\begin{array}{l}\text { Distribution } \\
\text { circulaire }\end{array}$ & X résultant & résultant $\mathrm{Zr}$ & résultant \\
\hline 5 & 0,119 & 0,191 & 0,119 & 0,119 & 0081 & 1,000 & 0,169 & 0,191 & 69,000 & 0,000 & 0,000 \\
\hline 6 & \multicolumn{2}{|c|}{ nb points discrétisation 32} & & & & Min pire cas & \begin{tabular}{|l|}
$\begin{array}{l}\text { Min } \\
\text { statistique }\end{array}$ \\
\end{tabular} & Max du point & Points & & \\
\hline 7 & & & & & & 0,119 & 0,169 & 0,1191 & $0,11,12,13,14,1$ & $15,16,17$ & \\
\hline 8 & & & & & & \begin{tabular}{|l|}
$\begin{array}{l}\text { Résultante } \\
\text { pire cas }\end{array}$ \\
\end{tabular} & $\begin{array}{l}\begin{array}{l}\text { Résultante } \\
\text { statistique }\end{array} \\
\end{array}$ & Nominal & \begin{tabular}{|l|}
$\begin{array}{l}\text { Résultante } \\
\text { pire cas }\end{array}$ \\
\end{tabular} & $\begin{array}{l}\text { Résultante } \\
\text { statistique }\end{array}$ & Nominal \\
\hline 9 & Surface/pièce & Maillons & & & Spécifications & 0,124 & 0,173 & 0,000 & 0,119 & 0,169 & 0,000 \\
\hline 10 & $8 \mathrm{a}$ & \multicolumn{3}{|c|}{ position d'une surface quelconque } & 519 & 0,005 & & 0,001 & 0,005 & & 0,001 \\
\hline 11 & $7 e+7 e+7 e+7 a$ & \multicolumn{3}{|c|}{\begin{tabular}{|l|l|} 
Jeu a/e virtuel position et orientation \\
|localisation
\end{tabular}} & $S 14+S 15+S 3+53$ & 0,063 & 0,024 & 0,043 & \begin{tabular}{|l|l|}
0,054 \\
\end{tabular} & 0,020 & 0,042 \\
\hline 12 & $1 \mathrm{e}$ & localisation & & & 513 & 0,007 & & 0,002 & \begin{tabular}{|l|}
0,007 \\
\end{tabular} & & 0,002 \\
\hline 13 & $4 c+4 c$ & \multicolumn{3}{|c|}{ localis ation+perpendicularité } & $523+524$ & 0,017 & & 0,005 & 0,017 & & 0,005 \\
\hline 14 & $17 c+17 c+8 e$ & \multirow{2}{*}{\multicolumn{2}{|c|}{\begin{tabular}{|l} 
Jeu e/c virtuel position \\
perpendicularité
\end{tabular}}} & & $527+514+516$ & 0,032 & & 0,023 & 0,032 & & 0,023 \\
\hline 15 & $3 a$ & & & & S2 & & & & 0,003 & 0,002 & 0,001 \\
\hline
\end{tabular}

Fig. 31. Plages de calcul de la résultante.

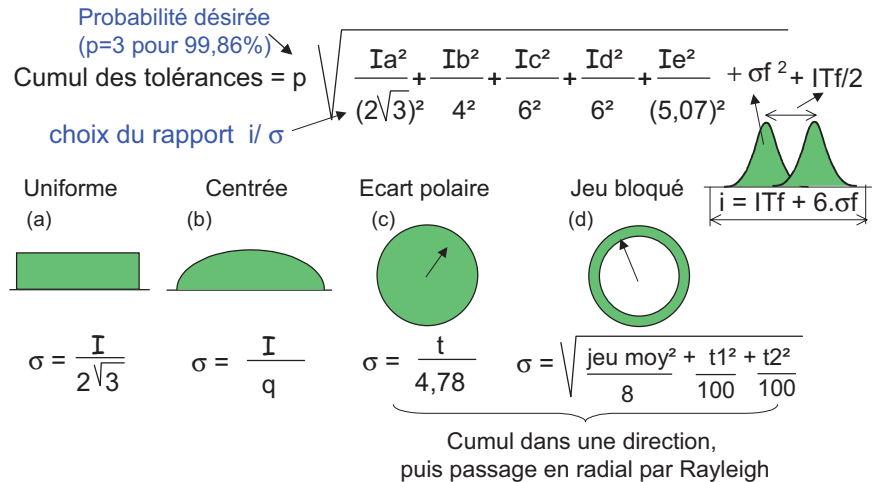

Fig. 32. Principe du calcul statistique.

La mobilité est toujours défavorable à l'exigence. Il faut donc toujours ajouter l'influence moyenne.

Influence moyenne : $e 4-a 1$, coef. : $0,93 \times(0,5+L / E)$ Écart type sur le jeu : $\sigma=\sqrt{\sigma 1 a^{2}+\sigma 6 e^{2}}$ coef. : $0,93 \times(0,5+L / E)$

Écart type sur l'influence de la position $(t 7 e)$ et de l'orientation $(t 8 e)$ : ces deux écarts polaires ne sont pas indépendants. L'influence maxi est : $I=(t 7 e+$ $t 8 c \times 2 . L / E)$. L'écart type d'une distribution polaire uniforme dans une direction donnée est $\sigma=I / 4,78$. La moyenne est 0 . Le coefficient est 0,93.

- $\mathrm{d}\left(S^{\mathrm{a}}, \overrightarrow{s a}\right)$ : donnée par la tolérance t9e. Moyenne 0 , écart type $\sigma 9 e$, coef. : 0,34.

$-\mathrm{d}\left(P^{\mathrm{e}}, \overrightarrow{p e}\right)$ : influence du défaut de plan supérieur de l'embout. L'influence est donnée figure 13c. $I=$ $t 9 c+t 7 c \times 2 . L / E$ (avec $E=$ étendue de la liaison axe/embout, $L=$ porte-à-faux de $P^{\mathrm{e}}$ d'où $L / E=2,43)$. L'influence de cet écart est polaire. L'écart type est $\sigma=I / 4,78$. La moyenne est 0 . Le coefficient est 0,34 .

- $\mathrm{d}\left(S^{\mathrm{e}}, \overrightarrow{s e}\right)$ : influence d'un jeu bloqué. Moyenne 0, écart type $\sigma=\sqrt{(c 2-e 2)^{2} / 8+t 3 c^{2} / 100+t 2 e^{2} / 100}$ coef. 0,93 .

\subsection{Calcul de la résultante}

Pour chaque point, il est donc possible de calculer la résultante maxi (ou mini) qui est définie pour une probabilité de respecter l'exigence.

$$
R_{\operatorname{maxi}}=\sum m_{i}+p \cdot \sqrt{\sum\left(\operatorname{coef}_{i} \cdot \sigma_{i}\right)^{2}}
$$

Le coefficient $p$ est donné dans la cellule G3. Il peut être déterminé à l'aide de la loi normale en fonction du risque accepté sur l'exigence. ( $p=3$ correspond à 99,97 \% de respect de l'exigence.) En effet, la distribution est quasi-normale s'il y a plus de 5 maillons indépendants dans la chaîne de cotes avec des intervalles d'influence du même ordre de grandeur. Dans les autres cas, le résultat donné est pessimiste, donc sans risque.

Pour une exigence radiale, la discrétisation donne des résultantes qui peuvent prendre les différentes distributions de la figure 33. La flèche représente la résultante maxi. Pour $p=3$, dans le cas (a), l'exigence a 0,03\% de risque d'être dépassé à ce point. Dans le cas (b), il y a deux fois plus de risque d'atteindre cette limite, il faut donc recalculer le coefficient p. Dans le cas (c), le risque existe pour tous les points. Il faut utiliser le modèle de Rayleigh pour calculer la probabilité globale (si les coordonnées $x$ et $y$ d'un point $M$ suivent des distributions normales d'écart type $\sigma x=\sigma y$, la distance $r$ du point $M$ à l'origine suit une distribution Rayleigh de moyenne $m=1,25 . \sigma x$ et d'écart type $\sigma r=0,655 . \sigma x)$. Dans le cas étudié, $\sigma x=\sqrt{\sum\left(\operatorname{coe} f_{i} \cdot \sigma_{i}\right)^{2}}$. La résultante est donnée par la relation :

$$
R_{\operatorname{maxi}}=\sum m_{i}+1,25 . \sigma x+p .0,655 . \sigma x
$$

Ce calcul est effectué dans la cellule H5.

\subsection{Problème des changements de contact}

Le cas de la figure 15 pose un dilemme non complètement résolu dans cette approche CLIC. En effet, 
(a) excentrée

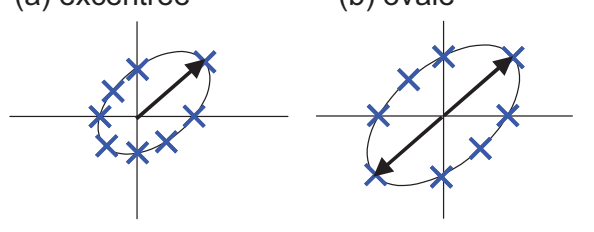

(c) circulaire

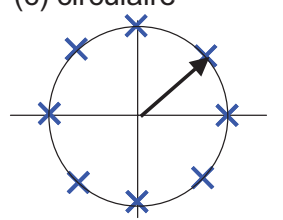

Fig. 33. Résultante radiale.

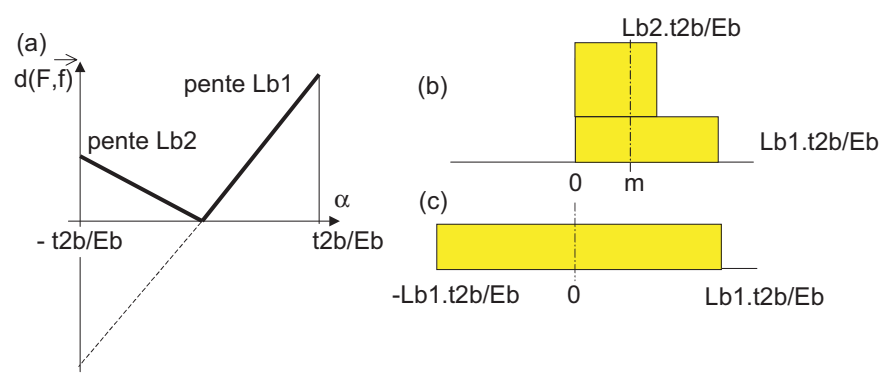

Fig. 34. Influence de l'angle.

les différentes configurations donnent des changements de points de contact en fonction des défauts angulaires. Cela se traduit par des courbes d'influence du type de la figure $34 \mathrm{a}$ avec une distribution théorique figure $34 \mathrm{~b}$. L'objectif du travail étant de déterminer le cas le plus défavorable, seule la partie droite de la distribution est significative. Dans ce but, la méthode CLIC cherche uniquement la situation la plus défavorable. Pour cela, on ne considère que la droite de pente $L b 1$ sur tout l'intervalle de variation de $\alpha$ (prolongement en pointillé), ce qui permet de travailler sur la distribution simple de la figure 34c. Par contre, cette simplification génère implicitement des cas virtuels sur la partie gauche qui pourraient compenser les autres composantes de la chaîne de cotes.

La prise en compte complète de tous les changements d'appui est en fait assez complexe, d'une part en raison du couplage primaire, secondaire, tertiaire, d'autre part lorsque le point de contact change de manière continue, par exemple sur le cercle qui entoure une face plane. Par contre, ce qui est très difficile c'est de définir la probabilité prévisionnelle de chaque configuration. Cette dernière remarque est également un lourd handicap pour toutes les méthodes de types Monte Carlo.

\section{Conclusion}

La méthode proposée comporte une première étape avec des algorithmes qui déterminent les spécifications à partir de la topologie de toutes les surfaces influentes de toutes les pièces. Dans un second temps, la discrétisation des surfaces tolérancées permet de trouver la relation au pire des cas donnant la résultante en chaque point en fonction des variations du nominal et des tolérances des différentes spécifications. Les écarts de position et angulaires peuvent alors être exprimés en fonction de tolérances fixées dans la première étape. Cette relation est décrite par un ensemble de formules EXCEL. Les données à manipuler sont tout à fait abordables et permettent d'envisager de traiter des mécanismes comportant un grand nombre d'exigences fonctionnelles. La simplicité obtenue permet de faire ultérieurement la synthèse des tolérances.

La méthode CLIC statistique proposée constitue un progrès significatif par rapport aux différentes méthodes pratiquées en entreprises. Toutefois, la difficulté reste l'estimation de la forme de la distribution à l'intérieur de l'intervalle d'influence. Pour une meilleure estimation, il faudrait connaître le processus détaillé de fabrication qui peut générer une probabilité plus forte au centre de l'intervalle d'influence. L'expérience montre que cette incertitude a finalement assez peu d'influence sur le résultat et qu'une hypothèse de distribution uniforme donne déjà d'excellants résultats.

Les résultats obtenus donnent les grandes tendances et permettent déjà de dégager des stratégies de répartition de tolérances qui peuvent permettre par exemple d'augmenter d'environ $40 \%$ les tolérances, dès qu'il y a plus de 5 pièces dans la chaîne de cotes et 100 \% lorsqu'il y a 10 pièces dans la chaîne de cote, le tout sans prendre de risque anormal en production, et sans nécessiter de suivi statistique des lots livrés, ce qui n'augmente pas le coût de contrôle.

\section{Références}

[1] B. Anselmetti, Cotation en localisation avec influence des contacts : méthode et optimisation, Revue internationale de CFAO et d'informatique Graphique 13 (1998) 27-42

[2] B. Anselmetti, Tolérancement, Méthode de cotation fonctionnelle, Éditions Hermes Sciences, Lavoisier, mai 2003

[3] P. Bourdet, E. Ballot, Géometrical behavior laws for computer aided tolerancing 4th CIRP seminar on Computer Aided Tolerancing, Tokyo, April 5-6 1995, Chapman Hill pp. 119-131

[4] P. Bourdet, A. Clément, A study of optimal-criteria identification based on small-displacement screw model, Annals of the CIRP 37 (1988) 503-506

[5] F. Luneau, F. Canal, P. Clozel, Tolérance 3D : Airbus applique la méthode $3 \mathrm{~A}$ avec un outil de chaîne de cotes $3 \mathrm{D}$, Revue Int. De CFAO et Informatique Graphique 18 (2003) $137-60$

[6] B. Anselmetti, generation of functional tolerancing based on positioning features, Computer Aided Design 38 (2006) 909-919

[7] R. Jayaraman, V. Srinivasan, Geometric tolerancing: I. virtual boundary requirements, IBM J. Res. Develop. 33 (1988) 90-104

[8] B. Anselmetti, K. Mawussi, H. Mejbri, Synthesis of tolerances starting from a fuzzy expression of the functional requirements, Colloque Int. CIRP Computer Aided Tolerancing, 24-25 avril 2001, Cachan, pp. 265-274

[9] B. Anselmetti, M. Radouani, Traitement statistique des chaînes de cotes radiales, Revue internationale de CFAO et d'informatique, Graphique 18 (2003) 61-75 